\title{
Stability of Highly Nonlinear Hybrid Stochastic Integro-differential Delay Equations
}

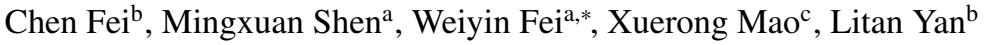 \\ ${ }^{a}$ School of Mathematics and Physics, Anhui Polytechnic University, Wuhu, Anhui 24100, China \\ ${ }^{b}$ Glorious Sun School of Business and Management, Donghua University, Shanghai 200051, China \\ ${ }^{c}$ Department of Mathematics and Statistics, University of Strathclyde, Glasgow G1 1XH, U.K.
}

\begin{abstract}
For the past few decades, the stability criteria for the stochastic differential delay equations (SDDEs) have been studied intensively. Most of these criteria can only be applied to delay equations where their coefficients are either linear or nonlinear but bounded by linear functions. Recently, the stability criterion for highly nonlinear hybrid stochastic differential equations is investigated in [Fei, Hu, Mao and Shen, Automatica, 2017]. In this paper, we investigate a class of highly nonlinear hybrid stochastic integro-differential delay equations (SIDDEs). First, we establish the stability and boundedness of hybrid stochastic integro-differential delay equations. Then the delay-dependent criteria of the stability and boundedness of solutions to SIDDEs are studied. Finally, an illustrative example is provided.
\end{abstract}

Keywords: stochastic integro-differential delay equation (SIDDE); nonlinear growth condition; asymptotic stability; Markovian switching; Lyapunov functional

\section{Introduction}

In many real-world systems, such as science, industry, economics and finance etc., we will encounter a time delay. The differential delay equations (DDEs) including the functional differential equations have been used to model such time-delay systems. Since the time-delay often 5 causes the instability of systems, stability of DDEs has been researched intensively for more than 50 years. Generally, the stability criteria are classified into the delay-dependent and delayindependent stability criteria. When the size of delays is incorporated into the delay-dependent stability criteria, the delay-dependent systems are generally less conservative than the delayindependent ones which work for any size of delays. There exists a very rich literature in this 10 area (see, e.g., [10, 11, 12, 16, 20]).

In 1980's, the stochastic differential delay equations were developed in order to model the real-world systems which are subject to external noises (see, e.g., [30]). Since then, in the study of SDDEs the stability has been one of the most important topics (see, e.g., [9, 19, 23, 24, 37, 38]).

${ }^{*}$ Corresponding author

Email address: wyf ei@ahpu.edu.cn (Weiyin Fei ) 
Since 1990's, the hybrid SDDEs (called also SDDEs with Markovian switching) were developed to model the real-world systems where they may experience abrupt changes in their parameters and structures in addition to uncertainties and time lags. One of the important issues in the study of hybrid SDDEs is the analysis of stability of control systems. Moreover, the delay-dependent stability criteria have been created by many authors (see, e.g., [26, 27, 28

20 29, 39, 40, 41, 2]). To our best knowledge, the existing delay-dependent stability criteria are mainly provided for the hybrid SDDEs where their coefficients are either linear or nonlinear but bounded by linear functions (or, satisfy the linear growth condition). Recently, [13, 14] initiate the investigation on the stability of the hybrid highly nonlinear stochastic delay differential equations. Based on the highly nonlinear hybrid SDDEs (see, e.g., [13, 14]), the stability of highly

25 nonlinear systems is further explored in [6, 7, 8, 34, 35]. However, the current states of many real systems depend on several history states of some time interval. Thus multiple time delay systems are also discussed (see, e.g., [21, 31]).

On the other hand, a real system depends on not only discrete delays (single or multiple ones) but also a whole history of states of the system with some lag interval. [4] considers a so nonhomogeneous Volterra integro-differential equation with the solution being a non-Markovian process. Moreover, the convergence and stability of the linear stochastic integro-differential delay equations were discussed in [1, 5, 15, 25, 17, 18, 32, 33, 36, 3]. With the energy of a hybrid system accumulated, a stable system might get unstable as it is disturbed by a white noise. In general, if a stable system has too long lag, then it might get unstable. Our problem ${ }_{35}$ is as follows: In how long lag time, the system can remain stable? To this end, we discuss an example as follows. Now we consider the stability of the following hybrid highly nonlinear SIDDE

$$
d X(t)= \begin{cases}\left(-10 X^{3}(t)-2 \hbar\left(X_{t}\right)\right) d t+\left(\hbar\left(X_{t}\right)\right)^{2} d B(t), & \text { if } i=1, \\ \left(\hbar\left(X_{t}\right)-5 X^{3}(t)\right) d t+\left(\hbar\left(X_{t}\right)\right)^{2} d B(t), & \text { if } i=2 .\end{cases}
$$

Here for $\tau \geq 0 X_{t}:=\{X(t+u):-\tau \leq u \leq 0\}, \hbar\left(X_{t}\right):=\frac{1}{\tau} \int_{-\tau}^{0} X(t+u) d u$ with $\hbar\left(X_{t}\right):=X(t)$ for $\tau=0, X(t) \in \mathbb{R}$ is the state of the highly non-linear hybrid system, $B(t)$ is a scalar Brownian motion, $r(t)$ is a Markovian chain with the state space $\mathbb{S}=\{1,2\}$ and its generator $\Gamma$ given by

$$
\Gamma=\left(\begin{array}{cc}
-1 & 1 \\
8 & -8
\end{array}\right)
$$

The above system (1.1) will switch from one mode to the other according to the probability law of the Markovian chain. If the time delay $\tau=0.01$, the computer simulation shows it is 40 asymptotically stable (see Figure 4.1). If the time-delay is large, say $\tau=3$, the computer simulation shows that the hybrid SIDDE (1.1) is unstable (see Figure 4.2 ). In other words, whether the hybrid SIDDE is stable or not depends on how small or large the time-delay is. On the other hand, both drift and diffusion coefficients of the hybrid SIDDE affect the stability of the systems due to highly nonlinear. However, there is no delay dependent criterion which can

45 be applied to the SIDDE to derive a sufficient bound on the time-delay $\tau$ such that the SIDDE is stable, although the stability criteria of the highly nonlinear hybrid SDDE have been discussed in [6] on the single delay. The aim of this paper is to establish the delay dependent criteria for the highly nonlinear hybrid SIDDEs.

Our main contributions are as follows: 
(a). The hybrid highly nonlinear stochastic integro-differential delay equations first are investigated, where the coefficients are highly nonlinear on both the current state $X(t)$ and the history $\hbar\left(X_{t}\right)$ with lag time $\tau \geq 0$.

(b). We established the theorem of the stability and boundedness of the solutions to the hybrid highly nonlinear SIDDEs similar to [13] where they only investigate the highly nonlinear hybrid 55 SDDE (see Theorem 2.4 in Section 2 below).

(c). The delay-dependent criteria are established first for the solutions to the hybrid highly nonlinear SIDDEs in Section 3.

(d). New mathematical techniques are well applied to solve our stability criteria, such as by constructing an appropriate Lyapunov functional.

\section{Notation and Assumptions}

Throughout this paper, unless otherwise specified, we use the following notation. If $A$ is a vector or matrix, its transpose is denoted by $A^{\top}$. If $x \in \mathbb{R}^{d}$, then $|x|$ is its Euclidean norm. For a matrix $A$, we let $|A|=\sqrt{\operatorname{trace}\left(A^{\top} A\right)}$ be its trace norm and $\|A\|=\max \{|A x|:|x|=1\}$ be the operator norm. Let $\mathbb{R}_{+}=[0, \infty)$. Denote by $C\left([-\tau, 0] ; \mathbb{R}^{d}\right)$ the family of continuous functions $\eta$ from $[-\tau, 0] \rightarrow \mathbb{R}^{d}$ with the norm $\|\eta\|=\sup _{-\tau \leq u \leq 0}|\eta(u)|$. If $A$ is a subset of $\Omega$, denote by $I_{A}$ its indicator function. Let $\left(\Omega, \mathcal{F},\left\{\mathcal{F}_{t}\right\}_{t \geq 0}, \mathbb{P}\right)$ be a complete probability space with a filtration $\left\{\mathcal{F}_{t}\right\}_{t \geq 0}$ satisfying the usual conditions. Let $B(t)=\left(B_{1}(t), \cdots, B_{m}(t)\right)^{\top}$ be an $m$-dimensional Brownian motion defined on the probability space. Let $r(t), t \geq 0$, be a right-continuous Markov chain on the probability space taking values in a finite state space $\mathbb{S}=\{1,2, \cdots, N\}$ with generator $\Gamma=\left(\gamma_{i j}\right)_{N \times N}$ given by

$$
\mathbb{P}\{r(t+\Delta)=j \mid r(t)=i\}= \begin{cases}\gamma_{i j} \Delta+o(\Delta) & \text { if } i \neq j, \\ 1+\gamma_{i i} \Delta+o(\Delta) & \text { if } i=j,\end{cases}
$$

where $\Delta>0$. Here $\gamma_{i j} \geq 0$ is the transition rate from $i$ to $j$ if $i \neq j$ while $\gamma_{i i}=-\sum_{j \neq i} \gamma_{i j}$. We assume that the Markov chain $r(\cdot)$ is independent of the Brownian motion $B(\cdot)$. Let

$$
\begin{aligned}
f(\cdot, \cdot, \cdot): \mathbb{R}^{d} \times \mathbb{S} \times \mathbb{R}_{+} \rightarrow \mathbb{R}^{d}, & g(\cdot, \cdot, \cdot): \mathbb{R}^{d} \times \mathbb{S} \times \mathbb{R}_{+} \rightarrow \mathbb{R}^{d \times m} \\
F(\hbar(\cdot), \cdot, \cdot): C\left([-\tau, 0] ; \mathbb{R}^{d}\right) \times \mathbb{S} \times \mathbb{R}_{+} \rightarrow \mathbb{R}^{d}, & G(\hbar(\cdot), \cdot, \cdot): C\left([-\tau, 0] ; \mathbb{R}^{d}\right) \times \mathbb{S} \times \mathbb{R}_{+} \rightarrow \mathbb{R}^{d \times m}
\end{aligned}
$$

be Borel measurable functions, where $\hbar(\phi):=\frac{1}{\tau} \int_{-\tau}^{0} \phi(u) d u, \phi \in C\left([-\tau, 0] ; \mathbb{R}^{d}\right)$ with $\hbar(\phi):=\phi(0)$ for $\tau=0$. Let the functional $X_{t}:=\{X(t+u):-\tau \leq u \leq 0\}$. Consider a $d$-dimensional hybrid highly nonlinear SIDDE with Assumption 2.1 below

$$
\begin{aligned}
d X(t)=[ & \left.f(X(t), r(t), t)+F\left(\hbar\left(X_{t}\right), r(t), t\right)\right] d t \\
& +\left[g(X(t), r(t), t)+G\left(\hbar\left(X_{t}\right), r(t), t\right)\right] d B(t)
\end{aligned}
$$

on $t \geq 0$ with initial data

$$
\{\eta(t):-\tau \leq t \leq 0\}=\eta \in C\left([-\tau, 0] ; \mathbb{R}^{d}\right), r(0)=i_{0} \in \mathbb{S} .
$$

The classical conditions for the existence and uniqueness of the global solution are the local Lipschitz condition and the linear growth condition (see, e.g., [24, 29]). In this paper, we need only the local Lipschitz condition. However, we will consider highly nonlinear hybrid SIDDEs which, in general, do not satisfy the linear growth condition in this paper. Therefore, we im65 pose the polynomial growth condition, instead of the linear growth condition. Let us state these conditions as an assumption for our aim. 
Assumption 2.1. Assume that for any $h>0$, there exists a positive constant $K_{h}$ such that

$$
\begin{aligned}
& |f(x, i, t)-f(\bar{x}, i, t)| \vee|g(x, i, t)-g(\bar{x}, i, t)| \\
& \vee|F(\hbar(\phi), i, t)-F(\hbar(\bar{\phi}), i, t)| \vee|G(\hbar(\phi), i, t)-G(\hbar(\bar{\phi}), i, t)| \leq K_{h}(|x-\bar{x}|+\|\phi-\bar{\phi}\|)
\end{aligned}
$$

for all $x, \bar{x}, \in \mathbb{R}^{d}$ and $\phi, \bar{\phi} \in C\left([-\tau, 0] ; \mathbb{R}^{d}\right)$ with $|x| \vee|\bar{x}| \vee\|\phi\| \vee\|\bar{\phi}\| \leq h$ and all $(i, t) \in \mathbb{S} \times \mathbb{R}_{+}$. Assume moreover that there exist three constants $K>0, q_{1} \geq 1$ and $q_{2} \geq 1$ such that

$$
\begin{aligned}
& |f(x, i, t)+F(\hbar(\phi), i, t)| \leq K\left(1+|x|^{q_{1}}+|\hbar(\phi)|^{q_{1}}\right) \\
& |g(x, i, t)+G(\hbar(\phi), i, t)| \leq K\left(1+|x|^{q_{2}}+|\hbar(\phi)|^{q_{2}}\right)
\end{aligned}
$$

for all $x \in \mathbb{R}^{d},(i, t) \in \mathbb{S} \times \mathbb{R}_{+}, \phi \in C\left([-\tau, 0] ; \mathbb{R}^{d}\right)$.

We emphasize that we are here interested in highly nonlinear SIDDEs which have either $q_{1}>1$ or $q_{2}>1$. We will refer condition (2.3) as the polynomial growth condition. It is known that Assumption 2.1 only guarantees that the SIDDE 2.1) with the initial data 2.2) has a unique maximal solution, which may explode to infinity at a finite time (see [24]). To avoid such a possible explosion, we need to impose an additional condition in terms of Lyapunov functions.

For our aim, we now give the following useful lemma.

Lemma 2.2. For nonnegative integers $m \geq k$, let the non-negative coefficient quasi-polynomial $U(x)=a_{m}|x|^{m}+\cdots+a_{k}|x|^{k}, x \in \mathbb{R}^{d}$, where $a_{i} \geq 0, i=k+1, \cdots, m-1$, and $a_{k}, a_{m}>0$. Assume that $x(t):[-\tau, \infty) \rightarrow \mathbb{R}^{d}$ is a continuous function, where $x(t)=\xi(t), t \in[-\tau, 0]$ and $\tau \geq 0$. Then, for any $\varepsilon \geq 0$, we have

$$
\begin{aligned}
& \int_{0}^{T} e^{\varepsilon t} U\left(\int_{-\tau}^{0} x(t+u) d u\right) d t \\
& \leq e^{\varepsilon \tau}\left(\int_{-\tau}^{0} e^{\varepsilon s} U(\xi(s)) d s+\int_{0}^{T} e^{\varepsilon s} U(x(s)) d s\right), \quad \forall T>0 .
\end{aligned}
$$

Proof. We first consider the case of $\tau>0$. Let $U(x)=|x|^{p}, p \geq 1$. For $p>1$, by the Hölder inequality with $q=\frac{p}{p-1}$, we obtain

$$
U\left(\frac{1}{\tau} \int_{-\tau}^{0} x(t+u) d u\right)=\left|\frac{1}{\tau} \int_{-\tau}^{0} x(t+u) d u\right|^{p} \leq \frac{1}{\tau} \int_{-\tau}^{0}|x(t+u)|^{p} d u .
$$

For $p=1$, the inequality above holds still. Therefore, we have

$$
\begin{aligned}
& \int_{0}^{T} e^{\varepsilon t}\left(\frac{1}{\tau} \int_{-\tau}^{0}|x(t+u)|^{p} d u\right) d t=\frac{1}{\tau} \int_{-\tau}^{0} \int_{0}^{T} e^{\varepsilon t}|x(t+u)|^{p} d t d u \\
& =\frac{1}{\tau} \int_{-\tau}^{0} \int_{u}^{T+u} e^{\varepsilon(s-u)}|x(s)|^{p} d s d u \leq \frac{1}{\tau} \int_{-\tau}^{0} \int_{-\tau}^{T} e^{\varepsilon s} e^{\varepsilon \tau}|x(s)|^{p} d s d u \\
& =e^{\varepsilon \tau} \int_{-\tau}^{T} e^{\varepsilon s}|x(s)|^{p} d s=e^{\varepsilon \tau}\left(\int_{-\tau}^{0} e^{\varepsilon s}|\xi(s)|^{p} d s+\int_{0}^{T} e^{\varepsilon s}|x(s)|^{p} d s\right) .
\end{aligned}
$$

Thus, we know, for $p=0,1, \cdots, m$,

$$
a_{p} \int_{0}^{T} e^{\varepsilon t}\left|\frac{1}{\tau} \int_{-\tau}^{0} x(t+u) d u\right|^{p} d t \leq e^{\varepsilon \tau}\left(\int_{-\tau}^{0} e^{\varepsilon s} a_{p}|\xi(s)|^{p} d s+\int_{0}^{T} e^{\varepsilon s} a_{p}|x(s)|^{p} d s\right),
$$

from which (2.4) holds. 
Next, for the case of $\tau=0$, the claim easily are obtained. Thus the proof is complete.

80 Let $C^{2,1}\left(\mathbb{R}^{d} \times \mathbb{S} \times \mathbb{R}_{+} ; \mathbb{R}_{+}\right)$denote the family of non-negative functions $U(x, i, t)$ defined on $(x, i, t) \in \mathbb{R}^{d} \times \mathbb{S} \times \mathbb{R}_{+}$which are continuously twice differentiable in $x$ and once in $t$. For such a function $U(x, i, t)$, let $U_{t}=\frac{\partial U}{\partial t}, U_{x}=\left(\frac{\partial U}{\partial x_{1}}, \cdots, \frac{\partial U}{\partial x_{d}}\right)$, and $U_{x x}=\left(\frac{\partial^{2} U}{\partial x_{k} \partial x_{l}}\right)_{d \times d}$. Let $C\left(\mathbb{R}^{d} \times\right.$ $[-\tau, \infty) ; \mathbb{R}_{+}$) denote the family of all continuous functions from $\mathbb{R}^{d} \times[-\tau, \infty)$ to $\mathbb{R}_{+}$. We can now state another assumption.

Assumption 2.3. Let $H(x), x \in \mathbb{R}^{d}$ be the nonnegative coefficient quasi-polynomial $H(\cdot)$ (see, Lemma 2.2). Assume that there exist the function $\bar{U} \in C^{2,1}\left(\mathbb{R}^{d} \times \mathbb{S} \times \mathbb{R}_{+} ; \mathbb{R}_{+}\right)$, and nonnegative constants $c_{0}, c_{1}, c_{2}$ and $q \geq 2\left(q_{1} \vee q_{2}\right)$ (where $q_{1}$ and $q_{2}$ are the same as in Assumption (2.1)) such that

$$
c_{2}<c_{1}, \quad|x|^{q} \leq \bar{U}(x, i, t) \leq H(x)
$$

for $\forall(x, i, t) \in \mathbb{R}^{d} \times \mathbb{S} \times \mathbb{R}_{+}$, and

$$
\begin{aligned}
& \mathbb{L} \bar{U}(x, \phi, i, t):=\bar{U}_{t}(x, i, t)+\bar{U}_{x}(x, i, t)(f(x, i, t)+F(\hbar(\phi), i, t)) \\
& +\frac{1}{2} \operatorname{trace}\left[(g(x, i, t)+G(\hbar(\phi), i, t))^{\top} \bar{U}_{x x}(x, i, t)(g(x, i, t)+G(\hbar(\phi), i, t))^{\top}\right]+\sum_{j=1}^{N} \gamma_{i j} \bar{U}(x, j, t) \\
& \leq c_{0}-c_{1} H(x)+c_{2} H(\hbar(\phi))
\end{aligned}
$$

${ }_{85}$ for all $x \in \mathbb{R}^{d},(i, t) \in \mathbb{S} \times \mathbb{R}_{+}, \phi \in C\left([-\tau, 0] ; \mathbb{R}^{d}\right)$.

In what follows, similar to the discussion in [13], we have the following theorem which shows the existence and uniqueness, the stability and boundedness of the global solution to highly nonlinear hybrid SIDDEs. In order to complete the proof of the theorem, we provide the existence and uniqueness of the maximal solution to SIDDE 2.1) delegated to Appendix A.

90 Theorem 2.4. Under Assumptions 2.1 and 2.3, the SIDDE (2.1) with initial data (2.2) has the following assertions:

(i) There is a unique global solution $X(t)$ to the $\operatorname{SIDDE}(2.1)$ on $t \in[-\tau, \infty)$.

(ii) The solution $X(t)$ obeys

$$
\limsup _{t \rightarrow \infty} \mathbb{E}|X(t)|^{q} \leq \frac{c_{0}}{\varepsilon}
$$

and

$$
\limsup _{t \rightarrow \infty} \frac{1}{t} \int_{0}^{t} \mathbb{E} H(X(s)) d s \leq \frac{c_{0}}{c_{1}-c_{2}},
$$

where $\varepsilon>0$ is the unique root to the equation

$$
c_{1}=\varepsilon+c_{2} e^{\varepsilon \tau}
$$

(iii) If, in addition, $c_{0}=0$, then the solution has the moment properties that

$$
\limsup _{t \rightarrow \infty} \frac{1}{t} \log \left(\mathbb{E}|X(t)|^{q}\right) \leq-\varepsilon
$$


and

$$
\int_{0}^{\infty} \mathbb{E} H(X(t)) d t \leq \frac{1}{c_{1}-c_{2}}\left(H(X(0))+\int_{-\tau}^{0} H(\eta(s)) d s\right) ;
$$

while it also has the sample (pathwise) properties that

$$
\limsup _{t \rightarrow \infty} \frac{1}{t} \log (|X(t)|) \leq-\frac{\varepsilon}{q} \quad \text { a.s. }
$$

and

$$
\int_{0}^{\infty} H(X(t)) d t<\infty \quad \text { a.s. }
$$

Proof: The whole proof is divided into three steps for three assertions.

Step 1. From Lemma A.3, we know that for the hybrid SIDDE 2.1) with the coefficients being locally Lipschitz continuous and any given initial data 2.2 , there is a unique maximal local solution $X(t)$ for $\forall t \in\left[-\tau, \sigma_{\infty}\right)$, where $\sigma_{\infty}$ is the explosion time. Let $m_{0}>0$ be sufficiently large for $m_{0} \geq\|\eta\|$. For each integer $m \geq m_{0}$, define the stopping time $\tau_{m}=\inf \left\{t \in\left[0, \sigma_{\infty}\right)\right.$ : $|X(t)| \geq m\}$, where, throughout this paper, $\inf \emptyset=\infty$. Clearly, $\tau_{m}$ is increasing as $m \rightarrow \infty$. Set $\tau_{\infty}=\lim _{m \rightarrow \infty} \tau_{m}$, whence $\tau_{\infty} \leq \sigma_{\infty}$ a.s. If we can show that $\tau_{\infty}=\infty$ a.s., then $\sigma_{\infty}=\infty$ a.s. and claim (i) follows. Next, we will show that $\tau_{\infty}=\infty$ a.s. By the generalized Itô formula (see, e.g., [29], Lemma 1.9 on p. 49) and condition (2.6), we can show that, for any $m \geq m_{0}$ and $t \geq 0$,

$$
\begin{aligned}
& \mathbb{E} \bar{U}\left(X\left(\tau_{m} \wedge t\right), r\left(\tau_{m} \wedge t\right), \tau_{m} \wedge t\right)-\bar{U}(X(0), r(0), 0) \\
& \leq \mathbb{E} \int_{0}^{\tau_{m} \wedge t}\left(c_{0}-c_{1} H(X(s))+c_{2} H\left(\hbar\left(X_{s}\right)\right)\right) d s
\end{aligned}
$$

from which, together with condition 2.5 , we have

$$
\begin{gathered}
\mathbb{E}\left|X\left(\tau_{m} \wedge t\right)\right|^{q} \leq H(X(0))+c_{0} t-c_{1} \mathbb{E} \int_{0}^{\tau_{m} \wedge t} H(X(s)) d s \\
+c_{2} \mathbb{E} \int_{0}^{\tau_{m} \wedge t} H\left(\hbar\left(X_{s}\right)\right) d s
\end{gathered}
$$

By Lemma 2.2, we derive

$$
\mathbb{E} \int_{0}^{\tau_{m} \wedge t} H\left(\hbar\left(X_{s}\right)\right) d s \leq \int_{-\tau}^{0} H(\eta(s)) d s+\mathbb{E} \int_{0}^{\tau_{m} \wedge t} H(X(s)) d s,
$$

which shows

$$
\left.\mathbb{E} \mid X\left(\tau_{m} \wedge t\right)\right)\left.\right|^{q} \leq K_{1}+c_{0} t-\left(c_{1}-c_{2}\right) \mathbb{E} \int_{0}^{\tau_{m} \wedge t} H(X(s)) d s,
$$

where $K_{1}=H(X(0))+c_{2} \int_{-\tau}^{0} H(\eta(s)) d s$. Noting that $c_{1}>c_{2}$, we get

$$
\mathbb{E}\left|X\left(\tau_{m} \wedge t\right)\right|^{q} \leq K_{1}+c_{0} t .
$$


Then we have $m^{q} \mathbb{P}\left(\tau_{m} \leq t\right) \leq K_{1}+c_{0} t$. Therefore, letting $m \rightarrow \infty$ in the inequality above, we 95 have $\mathbb{P}\left(\tau_{\infty} \leq t\right)=0$, which shows $\mathbb{P}\left(\tau_{\infty}>t\right)=1$. Due to arbitrariness of $t \geq 0$, we must have $\mathbb{P}\left(\tau_{\infty}=\infty\right)=1$ as required.

Step 2. By the generalized Itô formula, we obtain that for $t \geq 0$,

$$
\begin{aligned}
& \mathbb{E}\left(e^{\varepsilon\left(t \wedge \tau_{m}\right)} H\left(X\left(\tau_{m} \wedge t\right)\right)\right)-H(X(0)) \\
& \leq \mathbb{E} \int_{0}^{\tau_{m} \wedge t} e^{\varepsilon s}\left(c_{0}-\left(c_{1}-\varepsilon\right) H(X(s))+c_{2} H\left(\hbar\left(X_{s}\right)\right)\right) d s \\
& \leq \frac{c_{0}}{\varepsilon} e^{\varepsilon t}-\left(c_{1}-\varepsilon\right) \mathbb{E} \int_{0}^{t \wedge \tau_{m}} e^{\varepsilon s} H(X(s)) d s \\
& \quad+c_{2} \mathbb{E} \int_{0}^{t \wedge \tau_{m}} e^{\varepsilon s} H\left(\hbar\left(X_{s}\right)\right) d s .
\end{aligned}
$$

However, by Lemma 2.2, we derive

$$
\begin{aligned}
& \mathbb{E} \int_{0}^{\tau_{m} \wedge t} e^{\varepsilon s} H\left(\hbar\left(X_{s}\right)\right) d s \\
& \leq e^{\varepsilon \tau}\left(\int_{-\tau}^{0} e^{\varepsilon s} H(\eta(s)) d s+\mathbb{E} \int_{0}^{\tau_{m} \wedge t} e^{\varepsilon s} H(X(s)) d s\right),
\end{aligned}
$$

which, together with 2.97 and 2.15, shows that

$$
\mathbb{E}\left(e^{\varepsilon\left(t \wedge \tau_{m}\right)}\left|X\left(\tau_{m} \wedge t\right)\right|^{q}\right) \leq K_{2}+\frac{c_{0}}{\varepsilon} e^{\varepsilon t},
$$

where $K_{2}=H(X(0))+c_{2} e^{\varepsilon \tau} \int_{-\tau}^{0} e^{\varepsilon s} H(\eta(s)) d s$. Letting $m \rightarrow \infty$ we get that

$$
\mathbb{E}\left(e^{\varepsilon t}|X(t)|^{q}\right) \leq K_{2}+\frac{c_{0}}{\varepsilon} e^{\varepsilon t} .
$$

Thus, the desired claim 2.7 holds.

In order to show 2.8, from 2.14) we know that

$$
\left(c_{1}-c_{2}\right) \mathbb{E} \int_{0}^{\tau_{m} \wedge t} H(X(s)) d s \leq K_{1}+c_{0} t .
$$

Thus, letting $m \rightarrow \infty$ and using the Fubini theorem, we have

$$
\left(c_{1}-c_{2}\right) \mathbb{E} \int_{0}^{t} H(X(s)) d s \leq K_{1}+c_{0} t .
$$

Dividing both sides in 2.17) by $t$ and letting $t \rightarrow \infty$ we get the claim 2.8).

Step 3. Now we consider the case when $c_{0}=0$. From the calculations in the previous steps with $c_{0} \geq 0$, we know that they hold also for $c_{0}=0$. It then follows from 2.16 that

$$
\mathbb{E}|X(t)|^{q} \leq K_{2} e^{-\varepsilon t},
$$

which means the required claim 2.10. Moreover, from 2.17), we get

$$
\left(c_{1}-c_{2}\right) \mathbb{E} \int_{0}^{t} H(X(s)) d s \leq K_{1},
$$


which easily shows 2.11, which implies 2.13.

Finally, by the generalized Itô formula, we get that for any $t \geq 0$,

$$
\begin{aligned}
& e^{\varepsilon t} \bar{U}(X(t), r(t), t)-\bar{U}(X(0), r(0), 0) \\
& =\int_{0}^{t} e^{\varepsilon s}\left[\varepsilon \bar{U}(X(s), r(s), s)+\mathbb{L} \bar{U}\left(X(s), X_{s}, r(s), s\right)\right] d s+M(t),
\end{aligned}
$$

where $M(t)$ is a local martingale with the initial value $M(0)=0$. Due to Assumption 2.3 with $c_{0}=0$, we easily get in the same way as in Step 2 that

$$
e^{\varepsilon t}|X(t)|^{q} \leq K_{2}+M(t) .
$$

Using the non-negative semi-martingale convergence theorem (see, e.g., [22], Theorem 1.45 on p. 48), we get that

$$
\lim \sup _{t \rightarrow \infty}\left[e^{\varepsilon t}|X(t)|^{q}\right]<\infty \quad \text { a.s. }
$$

Thus, there exists a finite positive random variable $\zeta$ such that

$$
\sup _{0 \leq t<\infty}\left[e^{\varepsilon t}|X(t)|^{q}\right]<\zeta \quad \text { a.s. }
$$

which implies

$$
\limsup _{t \rightarrow \infty} \frac{1}{t} \log |X(t)|^{q} \leq-\varepsilon \quad \text { a.s. }
$$

Thus, the claim (2.12) holds. Hence we complete the proof.

\section{Delay-Dependent Asymptotic Stability of SIDDEs}

In this section, we will use the method of Lyapunov functionals to investigate the delaydependent asymptotic stability. We define two segments $\bar{X}_{t}:=\{X(t+s):-2 \tau \leq s \leq 0\}$ and $\bar{r}_{t}:=\{r(t+s):-2 \tau \leq s \leq 0\}$ for $t \geq 0$. For $\bar{X}_{t}$ and $\bar{r}_{t}$ to be well defined for $0 \leq t<2 \tau$, we set $X(s)=\eta(-\tau)$ for $s \in[-2 \tau,-\tau)$ and $r(s)=r_{0}$ for $s \in[-2 \tau, 0)$. We construct the Lyapunov functional as follows

$$
\begin{aligned}
V\left(\bar{X}_{t}, \bar{r}_{t}, t\right)=U( & X(t), r(t), t) \\
& +\theta_{1} \int_{-\tau}^{0} \int_{t+s}^{t}\left[\tau\left(1+\theta_{2}\right)\left|f(X(v), r(v), v)+F\left(\hbar\left(X_{v}\right), r(v), v\right)\right|^{2}\right. \\
& \left.+\left(1+\frac{1}{\theta_{2}}\right)\left|g(X(v), r(v), v)+G\left(\hbar\left(X_{v}\right), r(v), v\right)\right|^{2}\right] d v d s
\end{aligned}
$$

for $t \geq 0$, where $U \in C^{2,1}\left(\mathbb{R}^{d} \times \mathbb{S} \times \mathbb{R}_{+} ; \mathbb{R}_{+}\right)$such that

$$
\lim _{|x| \rightarrow \infty}\left[\inf _{(t, i) \in \mathbb{R}_{+} \times \mathbb{S}} U(x, t, i)\right]=\infty,
$$


and $\theta_{1}, \theta_{2}$ are positive numbers to be determined later while we set

$$
\begin{aligned}
& f(x, i, s)=f(x, i, 0), \quad g(x, i, s)=g(x, i, 0) \\
& F(\hbar(\phi), i, s)=F(\hbar(\phi), i, 0), \quad G(\hbar(\phi), i, s)=G(\hbar(\phi), i, 0)
\end{aligned}
$$

for all $x \in \mathbb{R}^{d},(i, s) \in \mathbb{S} \times[-2 \tau, 0), \phi \in C\left([-\tau, 0], \mathbb{R}^{d}\right)$. Applying the generalized Itô formula to $U(X(t), r(t), t)$, we get

$$
\begin{aligned}
& d U(X(t), r(t), t)=\left(U_{t}(X(t), r(t), t)\right. \\
& +U_{x}(X(t), r(t), t)\left(f(X(t), r(t), t)+F\left(\hbar\left(X_{t}\right), r(t), t\right)\right) \\
& +\frac{1}{2} \operatorname{trace}\left[\left(g(X(t), r(t), t)+G\left(\hbar\left(X_{t}\right), r(t), t\right)\right)^{\top}\right. \\
& \left.\quad \times U_{x x}(X(t), r(t), t)\left(g(X(t), r(t), t)+G\left(\hbar\left(X_{t}\right), r(t), t\right)\right)\right] \\
& \left.+\sum_{j=1}^{N} \gamma_{r(t), j} U(X(t), j, t)\right) d t+d M(t),
\end{aligned}
$$

for $t \geq 0$, where $M(t)$ is a continuous local martingale with $M(0)=0$. Denote an indicator function $\mathbb{1}_{[t-\tau, t]}(s)=1, s \in[t-\tau, t]$, or 0 . And define the functional $x \mathbb{1}_{[t-\tau, t]}:=\{\phi(s) ; \phi(s)=$ $x \mathbb{1}_{[t-\tau, t]}(s)=x, s \in[t-\tau, t]$, or 0$\}$ for $x \in \mathbb{R}^{d}$. Thus, rearranging terms give

$$
\begin{aligned}
d U(X(t), r(t), t) & \\
= & \left(U_{x}(X(t), r(t), t)\left[F\left(\hbar\left(X_{t}\right), r(t), t\right)-F\left(\hbar\left(X(t) \mathbb{1}_{[t-\tau, t]}\right), r(t), t\right)\right]\right. \\
& \left.+\mathcal{L} U\left(X(t), X_{t}, r(t), t\right)\right)+d M(t),
\end{aligned}
$$

where the function $\mathcal{L} U: \mathbb{R}^{d} \times C\left([-\tau, 0] ; \mathbb{R}^{d}\right) \times \mathbb{S} \times \mathbb{R}_{+} \rightarrow \mathbb{R}$ is defined by

$$
\begin{aligned}
& \mathcal{L} U(x, \phi, i, t)=U_{t}(x, i, t)+U_{x}(x, i, t)\left(f(x, i, t)+F\left(\hbar\left(x \mathbb{1}_{[t-\tau, t]}\right), i, t\right)\right) \\
& \quad+\frac{1}{2} \operatorname{trace}\left[(g(x, i, t)+G(\hbar(\phi), i, t))^{\top} U_{x x}(x, i, t)(g(x, i, t)+G(\hbar(\phi), i, t))\right]+\sum_{j=1}^{N} \gamma_{i j} U(x, j, t) .
\end{aligned}
$$

Moreover, the fundamental theory of calculus shows

$$
\begin{aligned}
d & \int_{-\tau}^{0} \int_{t+s}^{t}\left[\tau\left(1+\theta_{2}\right)\left|f(X(v), r(v), v)+F\left(\hbar\left(X_{v}\right), r(v), v\right)\right|^{2}\right. \\
& \left.\left.+\left(1+\frac{1}{\theta_{2}}\right)\left|g(X(v), r(v), v)+G\left(\hbar\left(X_{v}\right), r(v), v\right)\right|^{2}\right] d v d s\right) \\
= & \left(\tau \left[\tau\left(1+\theta_{2}\right)\left|f(X(v), r(v), v)+F\left(\hbar\left(X_{v}\right), r(v), v\right)\right|^{2}\right.\right. \\
& \left.+\left(1+\frac{1}{\theta_{2}}\right)\left|g(X(v), r(v), v)+G\left(\hbar\left(X_{v}\right), r(v), v\right)\right|^{2}\right] \\
& -\int_{t-\tau}^{t}\left[\tau\left(1+\theta_{2}\right)\left|f(X(v), r(v), v)+F\left(\hbar\left(X_{v}\right), r(v), v\right)\right|^{2}\right. \\
& \left.\left.+\left(1+\frac{1}{\theta_{2}}\right)\left|g(X(v), r(v), v)+G\left(\hbar\left(X_{v}\right), r(v), v\right)\right|^{2}\right] d v\right) d t .
\end{aligned}
$$


Lemma 3.1. With the notation above, $V\left(\bar{x}_{t}, \bar{r}_{t}, t\right)$ is an Itô process on $t \geq 0$ with its Itô differential

$$
d V\left(\bar{x}_{t}, \bar{r}_{t}, t\right)=L V\left(\bar{x}_{t}, \bar{r}_{t}, t\right) d t+d M(t),
$$

where $M(t)$ is a continuous local martingale with $M(0)=0$ and

$$
\begin{aligned}
L V\left(\bar{X}_{t}, \bar{r}_{t}, t\right)=U_{x}(X(t), r(t), t)\left[F\left(\hbar\left(X_{t}\right), r(t), t\right)\right. \\
\left.\quad-F\left(\hbar\left(X(t) \mathbb{1}_{[t-\tau, t]}\right), r(t), t\right)\right] \\
\quad+\mathcal{L} U\left(X(t), X_{t}, r(t), t\right) \\
\quad+\theta_{1} \tau\left[\tau\left(1+\theta_{2}\right)\left|f(X(t), r(t), t)+F\left(\hbar\left(X_{t}\right), r(t), t\right)\right|^{2}\right. \\
\left.\quad+\left(1+\frac{1}{\theta_{2}}\right)\left|g(X(t), r(t), t)+G\left(\hbar\left(X_{t}\right), r(t), t\right)\right|^{2}\right] \\
\quad-\theta_{1} \int_{t-\tau}^{t}\left[\tau\left(1+\theta_{2}\right)\left|f(X(v), r(v), v)+F\left(\hbar\left(X_{v}\right), r(v), v\right)\right|^{2}\right. \\
\left.\quad+\left(1+\frac{1}{\theta_{2}}\right)\left|g(X(v), r(v), v)+G\left(\hbar\left(X_{v}\right), r(v), v\right)\right|^{2}\right] d v .
\end{aligned}
$$

105

We here note that the expression for the martingale $M(t)$ in Lemma 3.1 is of no further use for our analysis, so it is not necessary to give the detailed expression. In what follows, to study the delay-dependent asymptotic stability of the SIDDE (2.1), we need to impose several new assumptions.

Assumption 3.2. Assume that there are functions $U \in C^{2,1}\left(\mathbb{R}^{d} \times \mathbb{S} \times \mathbb{R}_{+} ; \mathbb{R}_{+}\right)$, the nonnegative coefficient polynomial $U_{1} \in C\left(\mathbb{R}^{d} ; \mathbb{R}_{+}\right)$(see Lemma 2.2), and positive numbers $\alpha_{1}, \alpha_{2}$ and $\beta_{k}$ $(k=1,2,3)$ such that

$$
\alpha_{2}<\alpha_{1}
$$

and

$$
\begin{aligned}
& \mathcal{L} U(x, \phi, i, t)+\beta_{1}\left|U_{x}(x, i, t)\right|^{2} \\
& \quad+\beta_{2}|f(x, i, t)+F(\hbar(\phi), i, t)|^{2}+\beta_{3}|g(x, i, t)+G(\hbar(\phi), i, t)|^{2} \\
& \leq-\alpha_{1} U_{1}(x)+\alpha_{2} U_{1}(\hbar(\phi)),
\end{aligned}
$$

for all $x \in \mathbb{R}^{d},(i, t) \in \mathbb{S} \times \mathbb{R}_{+}, \phi \in C\left([-\tau, 0], \mathbb{R}^{d}\right)$.

Assumption 3.3. Assume that there exists a positive number $\varpi$ such that

$$
\left|F\left(\hbar\left(x \mathbb{1}_{[t-\tau, t]}\right), i, t\right)-F(\hbar(\phi), i, t)\right| \leq \varpi|x-\hbar(\phi)|
$$

110 for all $x \in \mathbb{R}^{d},(i, t) \in \mathbb{S} \times[-2 \tau, \infty), \phi \in C\left([-\tau, 0], \mathbb{R}^{d}\right)$.

Theorem 3.4. Let Assumptions 2.1 2.3, 3.2 and 3.3 hold. Assume also that

$$
\tau \leq \sup _{\theta_{2}>0}\left\{\left(\frac{4 \beta_{1} \beta_{2}}{\varpi^{2}\left(1+\theta_{2}\right)}\right)^{1 / 2} \bigwedge\left(\frac{4 \beta_{1} \beta_{3}}{\varpi^{2}\left(1+1 / \theta_{2}\right)}\right)\right\} .
$$

Then for any given initial data (2.2), the solution of the SIDDE (2.1) has the properties that

$$
\int_{0}^{\infty} \mathbb{E} U_{1}(X(t)) d t<\infty
$$


and

$$
\sup _{0 \leq t<\infty} \mathbb{E} U(X(t), r(t), t)<\infty .
$$

Proof: Fix the initial data $\eta \in C\left([-\tau, 0] ; \mathbb{R}^{d}\right)$ and $r_{0} \in \mathbb{S}$ arbitrarily. Let $k_{0}>0$ be a sufficiently large integer such that $\|\eta\|:=\sup _{-\tau \leq s \leq 0}|\eta(s)|<k_{0}$. For each integer $k>k_{0}$, define the stopping time

$$
\sigma_{k}=\inf \{t \geq 0:|X(t)| \geq k\} .
$$

It is easy to see that $\sigma_{k}$ is increasing as $k \rightarrow \infty$ and $\lim _{k \rightarrow \infty} \sigma_{k}=\infty$ a.s. By the generalized Itô formula we obtain from Lemma 3.1 that

$$
\mathbb{E} V\left(\bar{X}_{t \wedge \sigma_{k}}, \bar{r}_{t \wedge \sigma_{k}}, t \wedge \sigma_{k}\right)=V\left(\bar{X}_{0}, \bar{r}_{0}, 0\right)+\mathbb{E} \int_{0}^{t \wedge \sigma_{k}} L V\left(\bar{X}_{s}, \bar{r}_{s}, s\right) d s
$$

for any $t \geq 0$ and $k \geq k_{0}$. Let $\theta_{1}=\varpi^{2} /\left(4 \beta_{1}\right)$. By Assumption 3.3 and Cauchy-Schwartz inequality, it is easy to see that

$$
\begin{array}{r}
U_{x}(X(t), r(t), t)\left[F\left(\hbar\left(X(t) \mathbb{1}_{[t-\tau, t]}\right), r(t), t\right)-F\left(\hbar\left(X_{t}\right), r(t), t\right)\right] \\
\leq \beta_{1}\left|U_{x}(X(t), r(t), t)\right|^{2}+\frac{\varpi^{2}}{4 \beta_{1}}\left|X(t)-\frac{1}{\tau} \int_{-\tau}^{0} X(t+s) d s\right|^{2} \\
\quad \leq \beta_{1}\left|U_{x}(X(t), r(t), t)\right|^{2}+\frac{\varpi^{2}}{4 \beta_{1} \tau} \int_{-\tau}^{0}|X(t)-X(t+s)|^{2} d s .
\end{array}
$$

By condition 3.4, we also have

$$
\theta_{1} \tau^{2}\left(1+\theta_{2}\right) \leq \beta_{2} \quad \text { and } \quad \theta_{1} \tau\left(1+\frac{1}{\theta_{2}}\right) \leq \beta_{3} .
$$

It then follows from Lemma 3.1 that

$$
\begin{aligned}
L V\left(\bar{X}_{s}, \bar{r}_{s}, s\right) \leq & \mathcal{L} U(X(s), X(s), r(s), s)+\beta_{1}\left|U_{x}(X(s), r(s), s)\right|^{2} \\
& +\beta_{2}\left|f(X(s), r(s), s)+F\left(\hbar\left(X_{s}\right), r(s), s\right)\right|^{2} \\
& +\beta_{3}\left|g(X(s), r(s), s)+G\left(\hbar\left(X_{s}\right), r(s), s\right)\right|^{2} \\
& +\frac{\varpi^{2}}{4 \beta_{1} \tau} \int_{-\tau}^{0}|X(s)-X(s+v)|^{2} d v \\
& -\frac{\varpi^{2}}{4 \beta_{1}} \int_{s-\tau}^{s}\left[\tau\left(1+\theta_{2}\right)\left|f(X(v), r(v), v)+F\left(\hbar\left(X_{v}\right), r(v), v\right)\right|^{2}\right. \\
& \left.+\left(1+\frac{1}{\theta_{2}}\right)\left|g(X(v), r(v), v)+G\left(\hbar\left(X_{v}\right), r(v), v\right)\right|^{2}\right] d v .
\end{aligned}
$$

By Assumption 3.2, we then have

$$
\begin{aligned}
& L V\left(\bar{X}_{s}, \bar{r}_{s}, s\right) \leq-\alpha_{1} U_{1}(X(s))+\alpha_{2} U_{1}\left(\hbar\left(X_{s}\right)\right) \\
& \quad+\frac{\varpi^{2}}{4 \beta_{1} \tau} \int_{-\tau}^{0}|X(s)-X(s+v)|^{2} d v \\
& \quad-\frac{\varpi^{2}}{4 \beta_{1}} \int_{s-\tau}^{s}\left[\tau\left(1+\theta_{2}\right)\left|f(X(v), r(v), v)+F\left(\left(X_{v}\right), r(v), v\right)\right|^{2}\right. \\
& \left.\quad+\left(1+\frac{1}{\theta_{2}}\right)\left|g(X(v), r(v), v)+G\left(\hbar\left(X_{v}\right), r(v), v\right)\right|^{2}\right] d v .
\end{aligned}
$$


Substituting this into 3.6 implies

$$
\mathbb{E} V\left(\bar{X}_{t \wedge \sigma_{k}}, \bar{r}_{t \wedge \sigma_{k}}, t \wedge \sigma_{k}\right) \leq V\left(\bar{X}_{0}, \bar{r}_{0}, 0\right)+I_{1}+I_{2}-I_{3},
$$

where

$$
\begin{aligned}
I_{1}= & \mathbb{E} \int_{0}^{t \wedge \sigma_{k}}\left[-\alpha_{1} U_{1}(X(s))+\alpha_{2} U_{1}\left(\hbar\left(X_{s}\right)\right)\right] d s, \\
I_{2}= & \frac{\varpi^{2}}{4 \beta_{1} \tau} \mathbb{E} \int_{0}^{t \wedge \sigma_{k}} \int_{-\tau}^{0}|X(s)-X(s+v)|^{2} d v d s, \\
I_{3}= & \frac{\varpi^{2}}{4 \beta_{1}} \mathbb{E} \int_{0}^{t \wedge \sigma_{k}} \int_{s-\tau}^{s}\left[\tau\left(1+\theta_{2}\right)\left|f(X(v), r(v), v)+F\left(\hbar\left(X_{v}\right), r(v), v\right)\right|^{2}\right. \\
& \left.+\left(1+\frac{1}{\theta_{2}}\right)\left|g(X(v), r(v), v)+G\left(\hbar\left(X_{v}\right), r(v), v\right)\right|^{2}\right] d v d s .
\end{aligned}
$$

By Lemma 2.2(ii), we get that

$$
I_{1} \leq \alpha_{2} \int_{-\tau}^{0} U_{1}(\eta(v)) d v-\bar{\alpha} \mathbb{E} \int_{0}^{t \wedge \sigma_{k}} U_{1}(X(s)) d s,
$$

where $\bar{\alpha}=\alpha_{1}-\alpha_{2}>0$ by Assumption 3.2. Substituting this into 3.8. yields

$$
\bar{\alpha} \mathbb{E} \int_{0}^{t \wedge \sigma_{k}} U_{1}(X(s)) d s \leq C_{1}+I_{2}-I_{3},
$$

where $C_{1}$ is a constant defined by

$$
C_{1}=V\left(\bar{X}(0), \bar{r}_{0}, 0\right)+\alpha_{2} \int_{-\tau}^{0} U_{1}(\eta(s)) d s .
$$

Applying the classical Fatou lemma and let $k \rightarrow \infty$ in $(3.9)$ to obtain

$$
\bar{\alpha} \mathbb{E} \int_{0}^{t} U_{1}(X(s)) d s \leq C_{1}+\bar{I}_{2}-\bar{I}_{3},
$$

where

$$
\begin{aligned}
\bar{I}_{2}= & \frac{\varpi^{2}}{4 \beta_{1} \tau} \mathbb{E} \int_{0}^{t} \int_{-\tau}^{0}|X(s)-X(s+v)|^{2} d v d s, \\
\bar{I}_{3}= & \frac{\varpi^{2}}{4 \beta_{1}} \mathbb{E} \int_{0}^{t} \int_{s-\tau}^{s}\left[\tau\left(1+\theta_{2}\right)\left|f(X(v), r(v), v)+F\left(\hbar\left(X_{v}\right), r(v), v\right)\right|^{2}\right. \\
& \left.+\left(1+\frac{1}{\theta_{2}}\right)\left|g(X(v), r(v), v)+G\left(\hbar\left(X_{v}\right), r(v), v\right)\right|^{2}\right] d v d s .
\end{aligned}
$$

By the well-known Fubini theorem, we have

$$
\bar{I}_{2}=\frac{\varpi^{2}}{4 \beta_{1} \tau} \int_{0}^{t} \mathbb{E} \int_{-\tau}^{0}|X(s)-X(s+v)|^{2} d v d s .
$$


For $t \in[0, \tau]$, we have

$$
\begin{aligned}
\bar{I}_{2} & \leq \frac{\varpi^{2}}{2 \beta_{1} \tau} \int_{0}^{\tau} \int_{-\tau}^{0}\left(\mathbb{E}|X(s)|^{2}+\mathbb{E}|X(s+v)|^{2}\right) d v d s \\
& \leq \frac{\tau \varpi^{2}}{\beta_{1}}\left(\sup _{-\tau \leq v \leq \tau} \mathbb{E}|X(v)|^{2}\right) .
\end{aligned}
$$

For $t>\tau$, we have

$$
\bar{I}_{2} \leq \frac{\tau \varpi^{2}}{\beta_{1}}\left(\sup _{-\tau \leq v \leq \tau} \mathbb{E}|X(v)|^{2}\right)+\frac{\varpi^{2}}{4 \beta_{1} \tau} \int_{\tau}^{t} \mathbb{E} \int_{-\tau}^{0}|X(s)-X(s+v)|^{2} d v d s .
$$

Noting that, for $v \in[-\tau, 0]$,

$$
\begin{aligned}
& |X(s)-X(s+v)| \\
& =\mid \int_{s+v}^{s}\left(f(X(u), r(u), u)+F\left(\hbar\left(X_{u}\right), r(u), u\right)\right) d u \\
& \quad+\int_{s+v}^{s}\left(g(X(u), r(u), u)+G\left(\hbar\left(X_{u}\right), r(u), u\right)\right) d B(u) \mid .
\end{aligned}
$$

By using the inequality $(a+b)^{2} \leq\left(1+\theta_{2}\right) a^{2}+\left(1+\frac{1}{\theta_{2}}\right) b^{2}$ for the parameter choice, we get

$$
\begin{aligned}
& \mathbb{E}|X(s)-X(s+v)|^{2} \\
& \leq \mathbb{E} \int_{s+v}^{s}\left[\tau\left(1+\theta_{2}\right)\left|f(X(u), r(u), u)+F\left(\hbar\left(X_{u}\right), r(u), u\right)\right|^{2}\right. \\
& \left.\quad+\left(1+\frac{1}{\theta_{2}}\right)\left|g(X(u), r(u), u)+G\left(\hbar\left(X_{u}\right), r(u), u\right)\right|^{2}\right] d u .
\end{aligned}
$$

Thus we get

$$
\begin{aligned}
& \mathbb{E} \int_{-\tau}^{0}|X(s)-X(s+v)|^{2} d v \\
& \leq \mathbb{E} \int_{-\tau}^{0} \int_{s+v}^{s}\left[\tau\left(1+\theta_{2}\right)\left|f(X(u), r(u), u)+F\left(\hbar\left(X_{u}\right), r(u), u\right)\right|^{2}\right. \\
&\left.\quad+\left(1+\frac{1}{\theta_{2}}\right)\left|g(X(u), r(u), u)+G\left(\hbar\left(X_{u}\right), r(u), u\right)\right|^{2}\right] d u d v \\
& \leq \mathbb{E} \int_{-\tau}^{0} \int_{s-\tau}^{s}\left[\tau\left(1+\theta_{2}\right)\left|f(X(u), r(u), u)+F\left(\hbar\left(X_{u}\right), r(u), u\right)\right|^{2}\right. \\
&\left.\quad+\left(1+\frac{1}{\theta_{2}}\right)\left|g(X(u), r(u), u)+G\left(\hbar\left(X_{u}\right), r(u), u\right)\right|^{2}\right] d u d v \\
& \leq \tau \mathbb{E} \int_{s-\tau}^{s}\left[\tau\left(1+\theta_{2}\right)\left|f(X(u), r(u), u)+F\left(\hbar\left(X_{u}\right), r(u), u\right)\right|^{2}\right. \\
&\left.+\left(1+\frac{1}{\theta_{2}}\right)\left|g(X(u), r(u), u)+G\left(\hbar\left(X_{u}\right), r(u), u\right)\right|^{2}\right] d u .
\end{aligned}
$$


Notice also that

$$
\begin{aligned}
& \frac{1}{\tau} \int_{\tau}^{t} \mathbb{E} \int_{-\tau}^{0}|X(s)-X(s+v)|^{2} d v d s \\
& \leq \mathbb{E} \int_{\tau}^{t} \int_{s-\tau}^{s}\left[\tau\left(1+\theta_{2}\right)\left|f(X(u), r(u), u)+F\left(\hbar\left(X_{u}\right), r(u), u\right)\right|^{2}\right. \\
& \left.\quad+\left(1+\frac{1}{\theta_{2}}\right)\left|g(X(u), r(u), u)+G\left(\hbar\left(X_{u}\right), r(u), u\right)\right|^{2}\right] d u d s .
\end{aligned}
$$

Thus from 3.11) and 3.12 we get

$$
\bar{I}_{2} \leq \frac{\tau \varpi^{2}}{\beta_{1}}\left(\sup _{-\tau \leq v \leq \tau} \mathbb{E}|X(v)|^{2}\right)+\bar{I}_{3} .
$$

Together with (3.4), substituting 3.13 into 3.10 yields

$$
\bar{\alpha} \mathbb{E} \int_{0}^{t} U_{1}(X(s), s) d s \leq C_{1}+4 \beta_{3} \sup _{-\tau \leq v \leq \tau} \mathbb{E}|X(v)|^{2}:=C_{2} .
$$

Letting $t \rightarrow \infty$ gives

$$
\mathbb{E} \int_{0}^{\infty} U_{1}(X(s)) d s \leq \frac{C_{2}}{\bar{\alpha}} .
$$

Similarly, we see from 3.8 that

$$
\mathbb{E} U\left(X\left(t \wedge \sigma_{k}\right), r\left(t \wedge \sigma_{k}\right), t \wedge \sigma_{k}\right) \leq C_{1}+I_{2}-I_{3} .
$$

Letting $k \rightarrow \infty$ we get

$$
\mathbb{E} U(X(t), r(t), t) \leq C_{2}<\infty
$$

which shows

$$
\sup _{0 \leq t<\infty} \mathbb{E} U(X(t), r(t), t)<\infty
$$

Thus the proof is complete.

Corollary 3.5. Let the conditions of Theorem 3.4 hold. If there moreover exists a pair of positive constants $c$ and $p$ such that

$$
c|x|^{p} \leq U_{1}(x), \quad \forall x \in \mathbb{R}^{d},
$$

then for any given initial data (2.2), the solution of the SIDDE 2.1) satisfies

$$
\int_{0}^{\infty} \mathbb{E}|X(t)|^{p} d t<\infty
$$

That is, the SIDDE (2.1) is $H_{\infty}$-stable in $L^{p}$.

This corollary follows from Theorem 3.4 obviously. However, it does not follow from (3.14) that $\lim _{t \rightarrow \infty} \mathbb{E}|X(t)|^{p}=0$. 
Theorem 3.6. Let the conditions of Corollary 3.5 hold. If, moreover,

$$
p \geq 2 \text { and }\left(p+q_{1}-1\right) \vee\left(p+2 q_{2}-2\right) \leq q,
$$

then the solution of the SIDDE (2.1) satisfies

$$
\lim _{t \rightarrow \infty} \mathbb{E}|X(t)|^{p}=0
$$

115 for any initial data (2.2). That is, the SIDDE (2.1) is asymptotically stable in $L^{p}$.

Proof: Fix the initial data $(2.2)$ arbitrarily. For any $0 \leq t_{1}<t_{2}<\infty$, by the generalized Itô formula, we get

$$
\begin{aligned}
\mathbb{E} & \left|X\left(t_{2}\right)\right|^{p}-\mathbb{E}\left|X\left(t_{1}\right)\right|^{p} \\
= & \mathbb{E} \int_{t_{1}}^{t_{2}}\left(p|X(t)|^{p-2} X(t)^{\top}\left(f(X(t), r(t), t)+F\left(\hbar\left(X_{t}\right), r(t), t\right)\right)\right. \\
& +\frac{p}{2}|X(t)|^{p-2}\left|g(X(t), r(t), t)+G\left(\hbar\left(X_{t}\right), r(t), t\right)\right|^{2} \\
& +\frac{p(p-2)}{2}|X(t)|^{p-4} \mid\left(\left.X(t)^{\top}\left(g(X(t), r(t), t)+G\left(\hbar\left(X_{t}\right), r(t), t\right)\right)\right|^{2}\right) d t,
\end{aligned}
$$

which, due to Assumption 2.1. implies

$$
\begin{aligned}
& \left.|\mathbb{E}| X\left(t_{2}\right)\right|^{p}-\mathbb{E}\left|X\left(t_{1}\right)\right|^{p} \mid \\
& \leq \mathbb{E} \int_{t_{1}}^{t_{2}}\left(p|X(t)|^{p-1}\left|f(X(t), r(t), t)+F\left(\hbar\left(X_{t}\right), r(t), t\right)\right|\right. \\
& \left.\quad+\frac{p(p-1)}{2}|X(t)|^{p-2}\left|g(X(t), r(t), t)+G\left(\hbar\left(X_{t}\right), r(t), t\right)\right|^{2}\right) d t \\
& \leq \mathbb{E} \int_{t_{1}}^{t_{2}}\left(p K|X(t)|^{p-1}\left[1+|X(t)|^{q_{1}}+\left|\hbar\left(X_{t}\right)\right|^{q_{1}}\right]\right. \\
& \left.\quad+2 p(p-1) K^{2}|X(t)|^{p-2}\left[1+|X(t)|^{2 q_{2}}+\left|\hbar\left(X_{t}\right)\right|^{2 q_{2}}\right]\right) d t .
\end{aligned}
$$

By the inequalities,

$$
\begin{aligned}
|X(t)|^{p-1}\left\|\hbar\left(X_{t}\right)\right\|^{q_{1}} & \leq|X(t)|^{p+q_{1}-1}+\left\|\hbar\left(X_{t}\right)\right\|^{p+q_{1}-1}, \\
|X(t)|^{p-1} & \leq 1+|X(t)|^{q},
\end{aligned}
$$

etc., and noting that for any $1 \leq \bar{p} \leq q$, by the Hölder inequality and Theorem 2.4 we get

$$
\mathbb{E}\left|\hbar\left(X_{t}\right)\right|^{\bar{p}} \leq \sup _{-\tau \leq s \leq 0} \mathbb{E}|X(t+s)|^{\bar{p}} \leq\left(1+\sup _{-\tau \leq s<\infty} \mathbb{E}|X(s)|^{q}\right)<\infty,
$$

we can obtain

$$
\left.|\mathbb{E}| X\left(t_{2}\right)\right|^{p}-\mathbb{E}\left|X\left(t_{1}\right)\right|^{p} \mid \leq C_{3}\left(t_{2}-t_{1}\right)
$$

where

$$
C_{3}=4 p K(1+2(p-1) K)\left(1+\sup _{-\tau \leq t<\infty} \mathbb{E}|X(t)|^{q}\right)<\infty .
$$

Thus we have $\mathbb{E}|X(t)|^{p}$ is uniformly continuous in $t$ on $\mathbb{R}_{+}$. By 3.14$)$, there is a sequence $\left\{t_{l}\right\}_{l=1}^{\infty}$ in $\mathbb{R}_{+}$such that $\mathbb{E}\left|X\left(t_{l}\right)\right|^{p} \rightarrow 0$, which easily show the claim. Hence the proof is complete. 
Proposition 3.7. Let the conditions of Theorem 3.4 hold. Assume that there are positive constants $p$ and $c$ such that

$$
c|x|^{p} \leq U(x, i, t), \quad \forall(x, i, t) \in \mathbb{R}^{d} \times \mathbb{S} \times \mathbb{R}_{+} .
$$

Moreover assume there exists a function $W: \mathbb{R}^{d} \rightarrow \mathbb{R}_{+}$such that

$$
W(x)=0 \text { if and only if } x=0
$$

and

$$
W(x) \leq U_{1}(x), \forall x \in \mathbb{R}^{d} .
$$

Then for any given initial data (2.2), the solution $X(\cdot)$ to Eq. (2.1) obeys that

$$
\lim _{t \rightarrow \infty} X(t)=0 \quad \text { a.s. }
$$

Proof: Let $X(\cdot)$ be the solution to Eq. 2.1 with initial data $\eta$ defined in 2.2 . Since the conditions in Theorem 3.4 hold, by Fubini Theorem, we can show that

$$
C_{4}:=\int_{0}^{\infty} \mathbb{E} W(X(t)) d t<\infty,
$$

which implies

$$
\int_{0}^{\infty} W(X(t)) d t<\infty \quad \text { a.s. }
$$

Set $\sigma_{k}:=\inf \{t \geq 0:|X(t)|=k\}$. We observe from 3.16 that

$$
\lim _{t \rightarrow \infty} \inf W(X(t))=0 \quad \text { a.s. }
$$

Moreover, in the same way as Theorem 3.4 was proved, we can show that

$$
\mathbb{E}\left|X\left(T \wedge \sigma_{k}\right)\right|^{p} \leq C, \quad \forall T>0,
$$

which implies

$$
k^{p} \mathbb{P}\left(\sigma_{k} \leq T\right) \leq C
$$

Letting $T \rightarrow \infty$ yields

$$
k^{p} \mathbb{P}\left(\sigma_{k}<\infty\right) \leq C
$$

We now claim that

$$
\lim _{t \rightarrow \infty} W(X(t))=0 \quad \text { a.s. }
$$

In fact, if this is false, then we can find a number $\varepsilon \in(0,1 / 4)$ such that

$$
\mathbb{P}\left(\Omega_{1}\right) \geq 4 \varepsilon,
$$

where $\Omega_{1}=\left\{\lim \sup _{t \rightarrow \infty} W(X(t))>2 \varepsilon\right\}$. Recalling 3.18), we can find an integer $m$ sufficiently large for $\mathbb{P}\left(\sigma_{m}<\infty\right) \leq \varepsilon$. This means that

$$
P\left(\Omega_{2}\right) \geq 1-\varepsilon .
$$


where $\Omega_{2}:=\{|X(t)|<m$ for $\forall t \geq-\tau\}$. By 3.20 and 3.21 we get

$$
\mathbb{P}\left(\Omega_{1} \cap \Omega_{2}\right) \geq \mathbb{P}\left(\Omega_{1}\right)-\mathbb{P}\left(\Omega_{2}^{c}\right) \geq 3 \varepsilon,
$$

where $\Omega_{2}^{c}$ is the complement of $\Omega_{2}$. Let us now define the stopped process $\zeta(t)=X\left(t \wedge \sigma_{m}\right)$ for $t \geq-\tau$. Clearly, $\zeta(t)$ is a bounded Itô process with its differential

$$
d \zeta(t)=\varphi(t) d t+\psi(t) d B(t)
$$

where

$$
\begin{aligned}
& \varphi(t)=\left(f(X(t), r(t), t)+F\left(\hbar\left(X_{t}\right), r(t), t\right) I_{\left[0, \sigma_{m}\right)}(t),\right. \\
& \psi(t)=\left(g(X(t), r(t), t)+G\left(\hbar\left(X_{t}\right), r(t), t\right) I_{\left[0, \sigma_{m}\right)}(t),\right.
\end{aligned}
$$

where $f, g, F, G$ are defined by 2.17. Recalling the polynomial growth condition 2.3., we know that $\varphi(t)$ and $\psi(t)$ are bounded processes, say

$$
|\varphi(t)| \vee|\psi(t)| \leq C_{5} \quad \text { a.s. }
$$

for all $t \geq 0$ and some $C_{5}>0$. Moreover, we also observe that $|\zeta(t)| \leq m$ for all $t \geq-\tau$. Define a sequence of stopping times

$$
\begin{gathered}
\rho_{1}=\inf \{t \geq 0: W(\zeta(t)) \geq 2 \varepsilon\}, \\
\rho_{2 j}=\inf \left\{t \geq \rho_{2 j-1}: W(\zeta(t)) \leq \varepsilon\right\}, \quad j=1,2, \cdots, \\
\rho_{2 j+1}=\inf \left\{t \geq \rho_{2 j}: W(\zeta(t)) \geq 2 \varepsilon\right\}, \quad j=1,2, \cdots .
\end{gathered}
$$

From (3.17) and the definition of $\Omega_{1}$ and $\Omega_{2}$, we have

$$
\Omega_{1} \cap \Omega_{2} \subset\left\{\sigma_{m}=\infty\right\} \bigcap\left(\cap_{j=1}^{\infty}\left\{\rho_{j}<\infty\right\}\right) .
$$

We also note that for all $\omega \in \Omega_{1} \cap \Omega_{2}$, and $j \geq 1$,

$$
\begin{aligned}
& W\left(\zeta\left(\rho_{2 j-1}\right)\right)-W\left(\zeta\left(\rho_{2 j}\right)\right)=\varepsilon \quad \text { and } \\
& W(\zeta(t)) \geq \varepsilon \quad \text { when } t \in\left[\rho_{2 j-1}, \rho_{2 j}\right] .
\end{aligned}
$$

Since $W(\cdot)$ is uniformly continuous in the close ball $\bar{S}_{m}=\left\{x \in \mathbb{R}^{d}:|x| \leq m\right\}$. We can choose $\delta=\delta(\varepsilon)>0$ small sufficiently for which

$$
|W(\zeta)-W(\bar{\zeta})|<\varepsilon, \zeta, \bar{\zeta} \in \bar{S}_{m} \text {, with }|\zeta-\bar{\zeta}|<\delta .
$$

We emphasize that for $\omega \in \Omega_{1} \cap \Omega_{2}$, if $\left|\zeta\left(\rho_{2 j-1}+u\right)-\zeta\left(\rho_{2 j-1}\right)\right|<\delta$ for all $u \in[0, \lambda]$ and some $\lambda>0$, then $\rho_{2 j}-\rho_{2 j-1} \geq \lambda$. Choose a sufficiently small positive number $\lambda$ and then a sufficiently large positive integer $j_{0}$ such that

$$
2 C_{5}^{2} \lambda(\lambda+4) \leq \varepsilon \delta^{2} \text { and } C_{4}<\varepsilon^{2} \lambda j_{0} .
$$

By 3.20 and 3.22 , we can further choose a sufficiently large number $T$ for

$$
\mathbb{P}\left(\rho_{2 j_{0}} \leq T\right) \geq 2 \varepsilon .
$$


In particular, if $\rho_{2 j_{0}} \leq T$, then $\left|\zeta\left(\rho_{2 j_{0}}\right)\right|<m$, and hence $\rho_{2 j_{0}}<\sigma_{m}$ by the definition of $\zeta(t)$. We hence have

$$
\zeta(t, \omega)=X(t, \omega) \text { for all } 0 \leq t \leq \rho_{2 j_{0}} \text { and } \omega \in\left\{\rho_{2 j_{0}} \leq T\right\} .
$$

By the Burkholder-Davis-Gundy inequality, we can have that, for $1 \leq j \leq j_{0}$,

$$
\begin{aligned}
& \mathbb{E}\left(\sup _{0 \leq t \leq \lambda}\left|\zeta\left(\rho_{2 j-1} \wedge T+t\right)-\zeta\left(\rho_{2 j-1} \wedge T\right)\right|^{2}\right) \\
& \quad \leq 2 \mathbb{E}\left(\lambda \int_{\rho_{2 j-1} \wedge T}^{\rho_{2 j-1} \wedge T+\lambda}|\varphi(s)|^{2} d s+8 \mathbb{E} \int_{\rho_{2 j-1} \wedge T}^{\rho_{2 j-1} \wedge T+\lambda}|\psi(s)|^{2} d s\right) \\
& \quad \leq 2 C_{5}^{2} \lambda(\lambda+4),
\end{aligned}
$$

which, along with 3.26 and the Markov inequality, we can obtain that

$$
\mathbb{P}\left(\sup _{0 \leq t \leq \lambda}\left|\zeta\left(\rho_{2 j-1} \wedge T+t\right)-\zeta\left(\rho_{2 j-1} \wedge T\right)\right| \geq \delta\right) \leq \varepsilon .
$$

Noting that $\rho_{2 j-1} \leq T$ if $\rho_{2 j_{0}} \leq T$, we can derive from 3.27) and the above inequality that

$$
\begin{aligned}
& \mathbb{P}\left(\left\{\rho_{2 j_{0}} \leq T\right\} \cap\left\{\sup _{0 \leq t \leq \lambda}\left|\zeta\left(\rho_{2 j-1}+t\right)-\zeta\left(\rho_{2 j-1}\right)\right|<\delta\right\}\right) \\
&= \mathbb{P}\left(\rho_{2 j_{0}} \leq T\right) \\
& \quad-\mathbb{P}\left(\left\{\rho_{2 j_{0}} \leq T\right\} \cap\left\{\sup _{0 \leq t \leq \lambda}\left|\zeta\left(\rho_{2 j-1}+t\right)-\zeta\left(\rho_{2 j-1}\right)\right| \geq \delta\right\}\right) \\
& \geq \mathbb{P}\left(\rho_{2 j_{0}} \leq T\right)-\mathbb{P}\left(\sup _{0 \leq t \leq \lambda}\left|\zeta\left(\rho_{2 j-1}+t\right)-\zeta\left(\rho_{2 j-1}\right)\right| \geq \delta\right) \\
& \geq \varepsilon .
\end{aligned}
$$

This, together with 3.25, implies easily that

$$
\mathbb{P}\left(\left\{\rho_{2 j_{0}} \leq T\right\} \cap\left\{\rho_{2 j}-\rho_{2 j-1} \geq \lambda\right\}\right) \geq \varepsilon .
$$

By 3.24, and 3.28, we derive

$$
\begin{aligned}
C_{4} & \geq \sum_{j=1}^{j_{0}} \mathbb{E}\left(I_{\left\{\rho_{2 j_{0}} \leq T\right\}} \int_{\rho_{2 j-1}}^{\rho_{2 j}} W(X(t)) d t\right) \\
& \geq \varepsilon \sum_{j=1}^{j_{0}} \mathbb{E}\left(I_{\left\{\rho_{2 j_{0}} \leq T\right\}}\left(\rho_{2 j}-\rho_{2 j-1}\right)\right) \\
& \geq \varepsilon \lambda \sum_{j=1}^{j_{0}} \mathbb{P}\left(\left\{\rho_{2 j_{0}} \leq T\right\} \cap\left\{\rho_{2 j}-\rho_{2 j-1} \geq \lambda\right\}\right) \\
& \geq \varepsilon^{2} \lambda j_{0} .
\end{aligned}
$$

This contradicts the second inequality in (3.26). Thus $(3.19)$ must hold.

We now claim $\lim _{t \rightarrow \infty} X(t)=0$ a.s. If this were not true, then

$$
\varepsilon_{1}:=\mathbb{P}\left(\Omega_{3}\right)>0,
$$


where $\Omega_{3}=\left\{\limsup _{t \rightarrow \infty}|X(t)|>0\right\}$. On the other hand, by 3.18, we can find a positive integer $m_{0}$ large enough for $\mathbb{P}\left(\sigma_{m_{0}}<\infty\right) \leq 0.5 \varepsilon_{1}$. Let $\Omega_{4}=\left\{\sigma_{m_{0}}=\infty\right\}$. Then

$$
\mathbb{P}\left(\Omega_{3} \cap \Omega_{4}\right) \geq \mathbb{P}\left(\Omega_{3}\right)-\mathbb{P}\left(\Omega_{4}^{c}\right) \geq 0.5 \varepsilon_{1} .
$$

For any $\omega \in \Omega_{3} \cap \Omega_{4}, X(t, \omega)$ is bounded on $t \in \mathbb{R}_{+}$. We can then find a sequence $\left\{t_{j}\right\}_{j \geq 1}$ such that $t_{j} \rightarrow \infty$ and $X\left(t_{j}, \omega\right) \rightarrow \bar{X}(\omega) \neq 0$ as $j \rightarrow \infty$. This, together with the continuity of $W$, implies

$$
\lim _{j \rightarrow \infty} W\left(X\left(t_{j}, \omega\right)\right)=W(\bar{X}(\omega))>0,
$$

which show

$$
\limsup _{t \rightarrow \infty} W(X(t, \omega))>0 \text { for all } \omega \in \Omega_{3} \cap \Omega_{4} \text {. }
$$

But this contradicts 3.19). We therefore must have the assertion $\lim _{t \rightarrow \infty} X(t)=0$ a.s. Hence, the proof is complete.

\section{An Example for SIDDEs}

Let us now discuss an example to illustrate our theory.

Example 4.1. Let us consider the SIDDE (1.1) with the generator (1.2), we consider two case: $\tau=0.01$ and $\tau=2$ for all $t \geq 0$. In case with $\tau=0.01$, let the initial data $x(u)=2+\sin (u)$ for $u \in[-0.01,0], r(0)=2$, the sample paths of the Markov chain and the solution of the SIDDE are shown in Figure 4.1, which indicates that the SIDDE is asymptotically stable. In the case with $\tau=2$, let the initial data $x(u)=2+\sin (u)$ for $u \in[-3,0], r(0)=2$, the sample paths of the Markov chain and the solution of the affine delay SDE (1.1) are plotted in Figure 4.2, which indicates that the SIDDE is asymptotically unstable. From the example we can see SIDDE (1.1) is stable or not depends on how long or short the time-delay is.
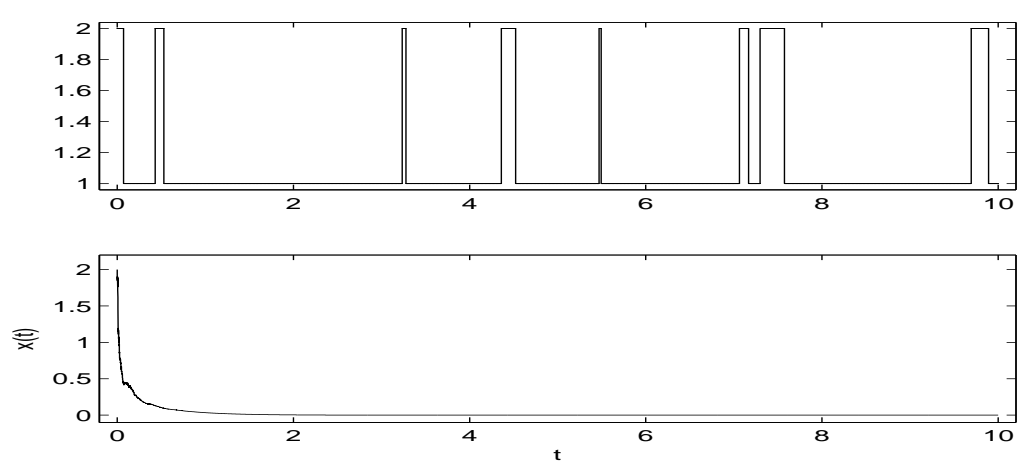

Figure 4.1: The computer simulation of the sample paths of the Markovian chain and the SIDDE (1.1) with $\tau=0.01$ using the Euler-Maruyama method with step size $10^{-3}$.

We can see coefficients defined by (1.1) satisfy Assumption 2.1 with $q_{1}=3$ and $q_{2}=2$. Define $\bar{U}(x, i, t)=|x|^{6}$ for $(x, i, t) \in \mathbb{R} \times \mathbb{S} \times \mathbb{R}_{+}$. It is easy to show that

$$
\mathbb{L} \bar{U}(x, \phi, i, t)=6 x^{5}(f(x, i, t)+F(\hbar(\phi), i, t))+15 x^{4}|g(x, i, t)+G(\hbar(\phi), i, t)|^{2}
$$



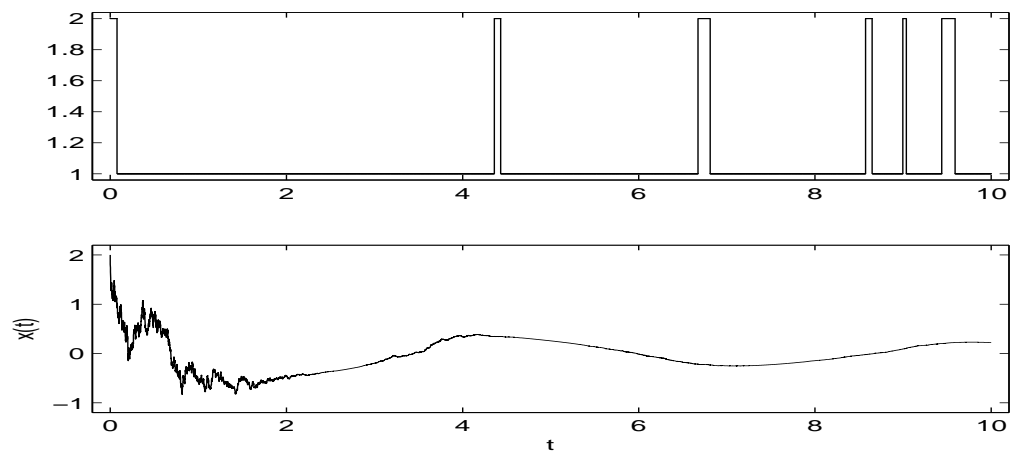

Figure 4.2: The computer simulation of the sample paths of the Markovian chain and the SIDDE 1.1] with $\tau=2$ using the Euler-Maruyama method with step size $10^{-3}$.

for $(x, i, t) \in \mathbb{R} \times \mathbb{S} \times \mathbb{R}_{+}, \phi \in C([-\tau, 0] ; \mathbb{R})$. Thus we get

$$
\begin{aligned}
\mathbb{L} \bar{U}(x, \phi, 1, t)= & 6 x^{5}\left(-10 x^{3}-2 \hbar(\phi)\right)+15 x^{4}|\hbar(\phi)|^{4} \\
& \leq 10 x^{6}+2|\hbar(\phi)|^{6}-52.5 x^{8}+7.5|\hbar(\phi)|^{8}
\end{aligned}
$$

and

$$
\begin{aligned}
\mathbb{L} \bar{U}(x, \phi, 2, t)= & 6 x^{5}\left(-5 x^{3}+\hbar(\phi)\right)+15 x^{4}|\hbar(\phi)|^{4} \\
& \leq 5 x^{6}+|\hbar(\phi)|^{6}-22.5 x^{8}+7.5|\hbar(\phi)|^{8} .
\end{aligned}
$$

Thus, we can obtain

$$
\begin{aligned}
\mathbb{L} \bar{U}(x, \phi, i, t) & \leq 10 x^{6}+2|\hbar(\phi)|^{6}-22.5 x^{8}+7.5|\hbar(\phi)|^{8} \\
& =\left(13+10 x^{6}+2|\hbar(\phi)|^{6}-1.5 x^{8}-0.5|\hbar(\phi)|^{8}\right)-21\left(1+x^{8}\right)+8\left(1+|\hbar(\phi)|^{8}\right) \\
& \leq c_{0}-c_{1}\left(1+x^{8}\right)+c_{2}\left(1+|\hbar(\phi)|^{8}\right),
\end{aligned}
$$

where

$$
0<c_{0}=\sup _{x \in \mathbb{R}, \phi \in C([-\tau, 0] ; \mathbb{R})}\left\{13+10 x^{6}+2|\hbar(\phi)|^{6}-1.5 x^{8}-0.5|\hbar(\phi)|^{8}\right\}<\infty
$$

and $H(x)=1+x^{8}$. Due to $c_{2}=8<c_{1}=21$, we know Assumption 2.3 holds. Therefore, Assumption 2.3 is satisfied. From Theorem 2.4, solution of the SIDDE (1.1) satisfies

$$
\sup _{-\tau \leq t<\infty} \mathbb{E}|X(t)|^{6}<\infty
$$

To verify Assumption 3.2 , we define

$$
U(x, i, t)=\left\{\begin{array}{cl}
x^{2}+x^{4}, & \text { if } i=1, \\
2 x^{2}+2 x^{4}, & \text { if } i=2
\end{array}\right.
$$


which shows

$$
U_{x}(x, i, t)= \begin{cases}2 x+4 x^{3}, & \text { if } i=1, \\ 4 x+8 x^{3}, & \text { if } i=2\end{cases}
$$

for $(x, i, t) \in \mathbb{R} \times \mathbb{S} \times \mathbb{R}_{+}$. By the equation [3.1, we have

$$
\begin{aligned}
\mathcal{L} U(x, \phi, 1, t)= & \left(2 x+4 x^{3}\right)\left(-10 x^{3}-2 x\right)+\frac{1}{2}|\hbar(\phi)|^{4}\left(2+12 x^{2}\right)+x^{2}+x^{4} \\
& \leq-3 x^{2}-27 x^{4}-38 x^{6}+|\hbar(\phi)|^{4}+4|\hbar(\phi)|^{6}
\end{aligned}
$$

and

$$
\begin{aligned}
\mathcal{L} U(x, \phi, 2, t)= & \left(4 x+8 x^{3}\right)\left(-5 x^{3}+x\right)+\frac{1}{2}|\hbar(\phi)|^{4}\left(4+24 x^{2}\right)-8 x^{2}-8 x^{4} \\
& \leq-4 x^{2}-20 x^{4}-36 x^{6}+2|\hbar(\phi)|^{4}+8|\hbar(\phi)|^{6} .
\end{aligned}
$$

Moreover

$$
\begin{gathered}
\left|U_{x}(x, i, t)\right|^{2}= \begin{cases}4 x^{2}+16 x^{4}+16 x^{6}, & \text { if } i=1, \\
16 x^{2}+64 x^{4}+64 x^{6}, & \text { if } i=2,\end{cases} \\
|f(x, i, t)+F(\hbar(\phi), i, t)|^{2}= \begin{cases}\left|10 x^{3}+2 \hbar(\phi)\right|^{2} \leq 200 x^{6}+8|\hbar(\phi)|^{2}, & \text { if } i=1, \\
\left|5 x^{3}+\hbar(\phi)\right|^{2} \leq 50 x^{6}+2|\hbar(\phi)|^{2}, & \text { if } i=2,\end{cases} \\
|g(x, i, t)+G(\hbar(\phi), i, t)|^{2}= \begin{cases}|\hbar(\phi)|^{4}, & \text { if } i=1, \\
|\hbar(\phi)|^{4}, & \text { if } i=2 .\end{cases}
\end{gathered}
$$

Setting $\beta_{1}=0.1, \beta_{2}=0.1, \beta_{3}=2$, using 44.2 - -4.4], we obtain that

$$
\begin{aligned}
& \mathcal{L} U(x, \phi, i, t)+\beta_{1}\left|U_{x}(x, i, t)\right|^{2}+\beta_{2}|f(x, i, t)+F(\hbar(\phi), i, t)|^{2}+\beta_{3}|g(x, i, t)+G(\hbar(\phi), i, t)|^{2} \\
& \leq \begin{cases}-2.6 x^{2}-25.4 x^{4}-16.4 x^{6}+0.8|\hbar(\phi)|^{2}+3|\hbar(\phi)|^{4}+4|\hbar(\phi)|^{6}, & \text { if } i=1, \\
-2.4 x^{2}-13.6 x^{4}-24.6 x^{6}+0.2|\hbar(\phi)|^{2}+4|\hbar(\phi)|^{4}+8|\hbar(\phi)|^{6}, & \text { if } i=2 .\end{cases}
\end{aligned}
$$

Thus, we get

$$
\begin{aligned}
& \mathcal{L} U(x, \phi, i, t)+\beta_{1}\left|U_{x}(x, i, t)\right|^{2}+\beta_{2}|f(x, i, t)+F(\hbar(\phi), i, t)|^{2}+\beta_{3}|g(x, i, t)+G(\hbar(\phi), i, t)|^{2} \\
& \leq-2.4 x^{2}-13.6 x^{4}-16.4 x^{6}+0.8|\hbar(\phi)|^{2}+4|\hbar(\phi)|^{4}+8|\hbar(\phi)|^{6} \\
& \leq-1.6\left(x^{2}+5 x^{4}+10 x^{6}\right)+0.8\left(|\hbar(\phi)|^{2}+5|\hbar(\phi)|^{4}+10|\hbar(\phi)|^{6}\right) \\
& \leq-1.6\left(x^{2}+5 x^{4}+10 x^{6}\right)+0.8\left(|\hbar(\phi)|^{2}+5|\hbar(\phi)|^{4}+10|\hbar(\phi)|^{6}\right) .
\end{aligned}
$$

Letting $U_{1}(x)=x^{2}+5 x^{4}+10 x^{6}$. Due to $\alpha_{1}=1.6, \alpha_{2}=0.8$, we get condition 3.2. In the case with $\tau=0$, the conditions in Theorem 3.4 obviously hold. Thus for each $\tau>0$, the conditions in Theorem 3.4 hold as well. Noting that $\varpi=2$ and taking $\theta_{2}=1$, we can obtain a upper bound of lag time $\tau \leq 0.07$. Thus, by Theorem 3.4, we can therefore conclude that the solution of the SIDDE (1.1) has the properties that

$$
\int_{0}^{\infty}\left(X^{2}(t)+X^{4}(t)+X^{6}(t)\right) d t<\infty \text { a.s. and } \int_{0}^{\infty} \mathbb{E}\left(X^{2}(t)+X^{4}(t)+X^{6}(t)\right) d t<\infty .
$$



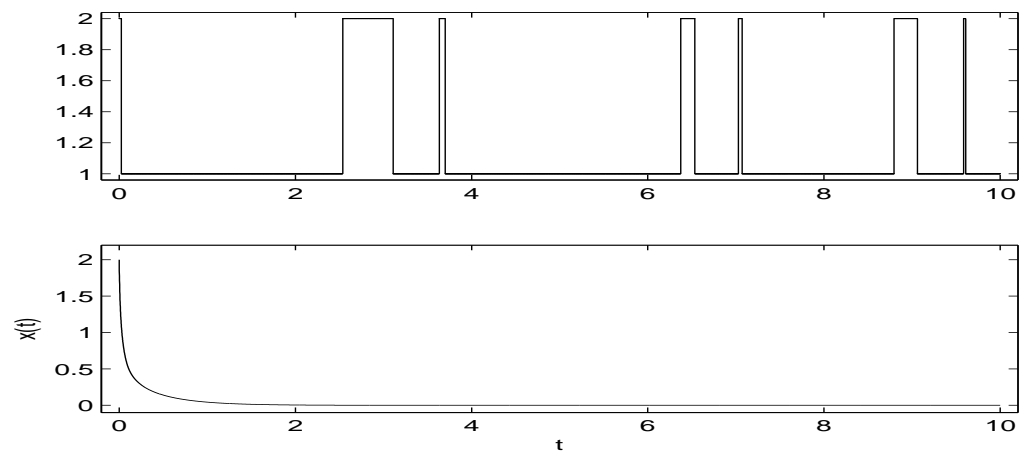

Figure 4.3 : The computer simulation of the sample paths of the Markovian chain and the SIDDE 1.1] with $\tau=0.07$ using the Euler-Maruyama method with step size $10^{-3}$.

Moreover, as $|X(t)|^{p} \leq x^{2}(t)+x^{4}(t)+x^{6}(t)$ for any $p \in[2,6]$, we have

$$
\int_{0}^{\infty} \mathbb{E}|X(t)|^{p} d t<\infty
$$

Recalling $q_{1}=3, q_{2}=2$ and $q=6$, we see that for $p=4$, all conditions of Theorem 3.6 are satisfied and hence we have

$$
\lim _{t \rightarrow \infty} \mathbb{E}|X(t)|^{4}=0 .
$$

We perform a computer simulation with the time-delay $\tau=0.07$ for all $t \geq 0$ and the initial data $x(u)=2+\sin (u)$ for $u \in[-0.07,0]$ and $r(0)=2$. The sample paths of the Markovian chain and the solution of the SIDDE (1.1) are plotted in Figure 4.3. The simulation supports our theoretical results.

\section{Conclusion}

In real applications, we are often faced with the stochastic integro-differential delay equations (SIDDEs). The boundedness and the stability of solutions to SIDDEs are the important topics. In this paper, we try to give the criteria of the stability and boundedness of the solutions to the hybrid highly nonlinear SIDDEs. To this end, we investigate the hybrid highly nonlinear hybrid SIDDEs. In fact, the stability of hybrid SDDEs have been studied for many years, most of the results in this topic require that the coefficients of equations are linear or nonlinear but bounded by linear functions. Recently, without the linear growth condition, [6] has established delay-dependent stability criteria for the highly nonlinear SDDEs by the method of Lyapunov function. In this paper, we first obtain the stability and boundedness of the hybrid highly nonlinear SIDDE in Section 2. In Section 3, by constructing a Lyapunov functional we further establish the delay-dependent stability criteria of the highly nonlinear SIDDEs, the $\mathrm{H}_{\infty}$ stability in $L^{p}$, and the asymptotic stability in $L^{p}$. Moreover, the almost surely asymptotic stability is also discussed. Finally, an illustrative example is given. 
Besides, it is noteworthy that (i) we give the Assumptions 2.1, 2.3, 3.2 and 3.3 only are the sufficient conditions which contain a kind of equations with their coefficients satisfying these assumptions; (ii) the condition (3.4) in Theorem 3.4 only tell us that the highly nonlinear integrodifferential system is stable as long as the system time lag $\tau$ verifies condition (3.4), but it cannot tell us if the system is stable as condition (3.4) is not verified. Thus, our delay boundary is conservative, and the less conservative delay boundary might be obtained. Moreover, we believe

155 that there exist other sufficient conditions such that the concerned systems are stable, which can be explored further in future.

\section{Appendix}

\section{A. The existence and uniqueness of the maximal solution}

To show the existence and uniqueness of the global solution to the SIDDE 2.1 with initial data 2.2, we first provide the following lemmas.

Lemma A.1. Assume that the condition (2.3) with $q_{1}=q_{2}=1$ holds, i.e. the coefficients of the SIDDE (2.1) satisfy the linear growth condition. If $X(\cdot)$ is a solution of the SIDDE (2.1), then we have

$$
\mathbb{E}\left(\sup _{0 \leq t \leq T}|X(t)|^{2}\right) \leq\left(1+3\|\eta\|^{2}\right) \exp \left(3 K^{2}(3 T+4) \max \left\{2,1+\|\eta\|^{2}\right\} T\right) .
$$

In particular, $X(\cdot) \in \mathcal{M}^{2}\left([0, T] ; \mathbb{R}^{d}\right)$.

Proof: For each integer $k \geq 1$, define the stopping time

$$
\tau_{k}=T \wedge \inf \{t \in[0, T]:|X(t)| \geq k\},
$$

which shows $\tau_{k} \uparrow T$ a.s. Thus $X_{k}(t):=X\left(t \wedge \tau_{k}\right)$ verifies

$$
\begin{aligned}
X_{k}(t)= & \eta(0)+\int_{0}^{t \wedge \tau_{k}}\left[f\left(X_{k}(s), r(s), s\right)+F\left(\hbar\left(X_{k, s}\right), r(s), s\right)\right] d s \\
& +\int_{0}^{t \wedge \tau_{k}}\left[g\left(X_{k}(s), r(s), s\right)+G\left(\hbar\left(X_{k, s}\right), r(s), s\right)\right] d B(s),
\end{aligned}
$$

where $X_{k, s}:=\left\{X_{k}(r): r \in[s-\tau, s]\right\}$. By condition $\left[2.3\right.$ with $q_{1}=q_{2}=1$ and the definition of $\hbar(\phi)$, we have

$$
\begin{aligned}
& \left|f\left(X_{k}(s), r(s), s\right)+F\left(\hbar\left(X_{k, s}\right), r(s), s\right)\right|^{2} \leq 3 K^{2}\left(1+\left|X_{k}(s)\right|^{2}+\sup _{s-\tau \leq r \leq s}\left|X_{k}(r)\right|^{2}\right), \\
& \left|g\left(X_{k}(s), r(s), s\right)+G\left(\hbar\left(X_{k, s}\right), r(s), s\right)\right|^{2} \leq 3 K^{2}\left(1+\left|X_{k}(s)\right|^{2}+\sup _{s-\tau \leq r \leq s}\left|X_{k}(r)\right|^{2}\right),
\end{aligned}
$$

where we have used $\left|\hbar\left(X_{k, s}\right)\right| \leq \| X_{k, s}\left|=\sup _{s-\tau \leq r \leq s}\right| X_{k}(r) \mid$. Thus, by employing the Hölder inequality and the Doob martingale inequality, we get that

$$
\begin{aligned}
\mathbb{E}\left(\sup _{0 \leq s \leq t}\left|X_{k}(s)\right|^{2}\right) & \leq 3\|\eta\|^{2}+3 t \mathbb{E} \int_{0}^{t}\left|f\left(X_{k}(v), r(v), v\right)+F\left(\hbar\left(X_{k, v}\right), r(v), v\right)\right|^{2} d v \\
& +3 \mathbb{E} \sup _{0 \leq s \leq t}\left|\int_{0}^{s \wedge \tau_{k}}\left[g\left(X_{k}(v), r(v), v\right)+G\left(\hbar\left(X_{k, v}\right), r(v), v\right)\right] d B(v)\right|^{2} \\
& \leq 3\|\eta\|^{2}+3 K^{2}(3 t+4) \mathbb{E} \int_{0}^{t}\left(1+\left|X_{k}(v)\right|^{2}+\sup _{v-\tau \leq r \leq v} \mid X_{k}(r) \|^{2}\right) d v .
\end{aligned}
$$


Noting $\sup _{v-\tau \leq r \leq v}\left|X_{k}(r)\left\|^{2} \leq\right\| \eta\left\|^{2}+\sup _{0 \leq r \leq v} \mid X_{k}(r)\right\|^{2}\right.$, we have

$$
1+\mathbb{E}\left(\sup _{0 \leq s \leq t}\left|X_{k}(s)\right|^{2}\right) \leq 1+3\|\eta\|^{2}+3 K^{2}(3 t+4) \max \left\{2,1+\|\eta\|^{2}\right\} \int_{0}^{t}\left(1+\mathbb{E} \sup _{0 \leq r \leq v} \mid X_{k}(r) \|^{2}\right) d v,
$$

From the Gronwall inequality, we know

$$
1+\mathbb{E}\left(\sup _{0 \leq s \leq t}\left|X_{k}(s)\right|^{2}\right) \leq\left(1+3\|\eta\|^{2}\right) \exp \left(3 K^{2}(3 t+4) \max \left\{2,1+\|\eta\|^{2}\right\} t\right),
$$

which shows A.1 by letting $k \rightarrow \infty$. Thus the proof is complete.

Lemma A.2. Let Assumption 2.1 with $q_{1}=q_{2}=1$ hold. Then there exists a unique solution $X(\cdot)$ to the SIDDE 2.1) and the solution belongs to $\mathcal{M}^{2}\left([0, T] ; \mathbb{R}^{d}\right)$.

Proof: We first give the proof of uniqueness. Let $X(\cdot)$ and $\tilde{X}(\cdot)$ be two solutions of the SIDDE 2.1] with the initial data 2.2]. From Lemma A.1 we know $X(\cdot), \tilde{X}(\cdot) \in \mathcal{M}^{2}\left([0, T] ; \mathbb{R}^{d}\right)$. Moreover, we have

$$
\begin{aligned}
& X(t)-\tilde{X}(t)=\int_{0}^{t}\left[(f(X(s), r(s), s)-f(\tilde{X}(s), r(s), s))+\left(F\left(\hbar\left(X_{s}\right), r(s), s\right)-F\left(\hbar\left(\tilde{X}_{s}\right), r(s), s\right)\right] d s\right. \\
& +\int_{0}^{t}\left[(g(X(s), r(s), s)-g(\tilde{X}(s), r(s), s))+\left(G\left(\hbar\left(X_{s}\right), r(s), s\right)-G\left(\hbar\left(\tilde{X}_{s}\right), r(s), s\right)\right)\right] d B(s) .
\end{aligned}
$$

For each $h \geq 1$, define the stopping time $\tilde{\tau}_{h}=\inf \{t \in[0, T]:|X(t)| \vee|\tilde{X}(t)| \geq h\}$. Obviously, $\tilde{\tau}_{h} \rightarrow T$ as $h \rightarrow \infty$. By the Hölder inequality, the Doob martingale inequality and the local Lipshcitz condition, we can show as in the proof of Lemma A.1 that

$$
\mathbb{E}\left(\sup _{0 \leq s \leq t}|X(s)-\tilde{X}(s)|^{2}\right) \leq C\left(K_{h}, \eta\right) \int_{0}^{t \wedge \tilde{\tau}_{h}} \mathbb{E}\left(\sup _{0 \leq r \leq v}|X(r)-\tilde{X}(r)|^{2}\right) d v .
$$

From the Gronwall inequality, we get

$$
\mathbb{E}\left(\sup _{0 \leq s \leq t \wedge \tilde{\tau}_{h}}|X(s)-\tilde{X}(s)|^{2}\right)=0 .
$$

Letting $h \rightarrow \infty$, we know that $X(t)=\tilde{X}(t)$ for all $0 \leq t \leq T$ almost surely. Thus the uniqueness has been proved.

Next the proof of existence is similar to that of [29, Theorem 3.13, page 89]. Due to page limit, we omit the details.

The following assertion shows the existence of unique maximal local solution under the local Lipschitz condition without the linear growth condition similar to the discussion in [29, Theorem 3.15 , page 91].

Lemma A.3. Let Assumption 2.1 hold. Then there exists a unique maximal local solution to the SIDDE 2.1] with the initial data [2.2.

Proof: By a truncation procedure, we show our claim. For each integer $m \geq 1$, define the truncation functions

$$
\begin{array}{r}
f_{m}(x, i, t)=\left\{\begin{array}{lll}
f(x, i, t) & \text { if } & |x| \leq m, \\
f(m x /|x|, i, t) & \text { if } & |x|>m,
\end{array}\right. \\
F_{m}(\hbar(\phi), i, t)=\left\{\begin{array}{lll}
F(\hbar(\phi), i, t) & \text { if } & \|\phi\| \leq m, \\
F(m \hbar(\phi) /\|\phi\|, i, t) & \text { if } & \|\phi\|>m,
\end{array}\right.
\end{array}
$$


where $x \in \mathbb{R}^{d}$ and $\phi \in C\left([-\tau, 0] ; \mathbb{R}^{d}\right)$. Similarly, we can define the functions $g_{m}(x, i, t)$ and $G_{m}(\hbar(\phi), i, t)$. Due to $|\hbar(\phi)| \leq\|\phi\|$, we can know that the functions $f_{m}, F_{m}, g_{m}$ and $G_{m}$ satisfy Lipschitz condition and the linear growth condition. Hence from Lamma A.2, there exists a unique solution $X_{m}(\cdot)$ in $\mathcal{M}^{2}\left([0, T] ; \mathbb{R}^{d}\right)$ to the equation

$$
\begin{aligned}
d X_{m}(t)= & {\left[f_{m}\left(X_{m}(t), r(t), t\right)+F_{m}\left(\hbar\left(X_{m, t}\right), r(t), t\right)\right] d t } \\
& +\left[g_{m}\left(X_{m}(t), r(t), t\right)+G_{m}\left(\hbar\left(X_{m, t}\right), r(t), t\right)\right] d B(t)
\end{aligned}
$$

on $t \geq 0$ with initial data 2.2. Define the stopping time

$$
v_{m}=T \wedge \inf \left\{t \in[0, T]:\left|X_{m}(t)\right| \geq m\right\} .
$$

We easily know

$$
X_{m}(t)=X_{m+1}(t) \quad \text { if } \quad 0 \leq t \leq v_{m},
$$

which means that $v_{m}$ is increasing, and $v_{\infty}=\lim _{m \rightarrow \infty} v_{m}$. Define now the process $\{X(t): 0 \leq t<$ $\infty$ by

$$
X(t)=X_{m}(t), v_{m-1} \leq t<v_{m}, m \geq 1,
$$

where $v_{0}=0$. In virtue of A.4 , we have $X\left(t \wedge v_{m}\right)=X_{m}\left(t \wedge v_{m}\right)$. Thus from A.3, we get

$$
\begin{aligned}
d X\left(t \wedge v_{m}\right) & =\eta(0)+\int_{0}^{t \wedge v_{m}}\left[f_{m}(X(s), r(s), s)+F_{m}\left(\hbar\left(X_{s}\right), r(s), s\right)\right] d s \\
& +\int_{0}^{t \wedge v_{m}}\left[g_{m}(X(s), r(s), s)+G_{m}\left(\hbar\left(X_{s}\right), r(s), s\right)\right] d B(s) \\
& =\eta(0)+\int_{0}^{t \wedge v_{m}}\left[f(X(s), r(s), s)+F\left(\hbar\left(X_{s}\right), r(s), s\right)\right] d s \\
& +\int_{0}^{t \wedge v_{m}}\left[g(X(s), r(s), s)+G\left(\hbar\left(X_{s}\right), r(s), s\right)\right] d B(s)
\end{aligned}
$$

for each $t \in[0, T]$ and $m \geq 1$. It is easy to verify that if $v_{\infty}<T$, then

$$
\limsup _{t \rightarrow v_{\infty}}|X(t)| \geq \limsup _{m \rightarrow \infty} X\left(v_{m}\right)=\limsup _{m \rightarrow \infty} X_{m}\left(v_{m}\right)=\infty .
$$

Therefore, $\left\{X(t): 0 \leq t<v_{\infty}\right\}$ is a maximal local solution. Now we show the uniqueness. To this end, let $\left\{\tilde{X}(t): 0 \leq t<\tilde{v}_{\infty}\right\}$ be another maximal local solution. Define $\tilde{v}_{m}=\tilde{v}_{\infty} \wedge \inf \{t \in$ $\left[\left[0, \tilde{v}_{\infty}\left[[:|\tilde{X}(t)| \geq m\}\right.\right.\right.$. We can show that $\tilde{v}_{m} \rightarrow \tilde{v}_{\infty}$ a.s. and

$$
\mathbb{P}\left\{X(t)=\tilde{X}(t) \forall t \in\left[\left[0, v_{m} \wedge \tilde{v}_{m}[[\}=1 \quad \forall m \geq 1 .\right.\right.\right.
$$

Letting $m \rightarrow \infty$, we get

$$
\mathbb{P}\left\{X(t)=\tilde{X}(t) \forall t \in\left[\left[0, v_{\infty} \wedge \tilde{v}_{\infty}[[\}=1 .\right.\right.\right.
$$

Next, we need to prove that $v_{\infty}=\tilde{v}_{\infty}$ a.s. Indeed, for almost any $\omega \in\left\{v_{\infty}<\tilde{v}_{\infty}\right\}$, we can obtain

$$
\left|\tilde{X}\left(v_{\infty}, \omega\right)\right|=\lim _{m \rightarrow \infty}\left|\tilde{X}\left(v_{m}, \omega\right)\right|=\lim _{m \rightarrow \infty}\left|X\left(v_{m}, \omega\right)\right|=\infty
$$

which contracts the fact that $\tilde{X}(t, \omega)$ is continuous on $t \in\left[0, \tilde{v}_{\infty}(\omega)\right)$. Thus we must have $v_{\infty} \geq \tilde{v}_{\infty}$ a.s. Similarly, we can show $v_{\infty} \leq \tilde{v}_{\infty}$ a.s. Hence we get $v_{\infty}=\tilde{v}_{\infty}$ a.s. Thus the proof is complete. 


\section{Acknowledgements}

Many helpful comments and suggestions from the editor and referees are gratefully acknowledged. The authors would like to thank Liangjian Hu and Wei Liu for their helpful discussions.

180 This paper is supported by the Natural Science Foundation of China (71571001), China Scholarship Council (201806630049), the Promoting Plan of Higher Education of Anhui Province (TSKJ2016B11), the Royal Society (WM160014, Royal Society Wolfson Research Merit Award), the Royal Society and the Newton Fund (NA160317, Royal Society- Newton Advanced Fellowship) and the EPSRC (EP/K503174/1) for their financial support.

\section{References}

[1] J.M. Benker, G. Pap, Asymptotic inference for a stochastic differential equation with uniformly distributed time delay, J. Stat. Plan. Infer. 167(2015)182-192.

[2] P. Balasubramaniam, R. Krishnasamy, R. Rakkiyappan, Delay-interval-dependent robust stability results for uncertain stochastic systems with Markovian jumping parameters, Nonlinear Anal.-Hybri. Syst. 5(2011)681-691.

[3] S. Cong, On almost sure stability conditions of linear switching stochastic differential systems, Nonlinear Anal.Hybri. Syst. 22(2016)108-115.

[4] H.M. Dietz, A non-Markovian relative to the Ornstein-Uhlenbeck process and some of its local statistical properties, Scand. J. Stat. 19(1992)363-379.

[5] X.H. Ding, K.N. Wu, M.Z. Liu , Convergence and stability of the semi-implicit Euler method for linear stochastic differential intgro-differential equations, Int. J. Comput. Math. 83(2006)753-763.

[6] W. Fei, L. Hu, X. Mao, M. Shen, Delay dependent stability of highly nonlinear hybrid stochastic systems, Automatica 28(2017)165-170

[7] W. Fei, L. Hu, X. Mao, M. Shen, Generalised criteria on delay dependent stability of highly nonlinear hybrid stochastic systems, Submitted to International Journal of Robust and Nonlinear Control.

200 [8] W. Fei, L. Hu, X. Mao, M. Shen, Structured robust stability and boundedness of nonlinear hybrid delay systems, SIAM J. Control Optim. 56 (4) (2018) 2662-2689.

[9] M. Frederic, Stability analysis of time-varying neutral time-delay systems, IEEE Trans. Automat. Control 60(2016)540-546

[10] E. Fridman, Introduction to Time-Delay Systems: Analysis and Control, Birkhauser, 2014.

205 [11] A. Garab, V. Kovács, T. Krisztin, Global stability of a price model with multiple delays, Discrete \& Continuous Dynamical Systems- Series A 36(12)(2016)6855-6871.

[12] J.K. Hale, S.M. Lunel, Introduction to Functional Differential Equations, Springer-Verlag, 1993.

[13] L. Hu, X. Mao, Y. Shen, Stability and boundedness of nonlinear hybrid stochastic differential delay equations, Systems \& Control Letters 62(2013)178-187.

210 [14] L. Hu, X. Mao, L. Zhang, Robust stability and boundedness of nonlinear hybrid stochastic delay equations, IEEE Trans. Automat. Control 58(9)(2013)2319-2332.

[15] P. Hu, C.M. Huang, Stability of stochastic $\theta$-methods for stochastic delay integro-differential equations, Int. J. Comput. Math. 88(7)(2011)1417-1429.

[16] V.B. Kolmanovskii, V.R. Nosov, Stability of Functional Differential Equations, Academic Press, 1986.

215 [17] U. Küchler, M. Sørensen, A simple estimator for discrete-time samples from affine stochastic delay differential equations, Statistical Inference for Stochastic Processes 13(2010)125-132.

[18] U. Küchler, M. Sørensen, Statistical inference for discrete-time samples from affine stochastic delay differential equations, Bernoulli 19(2)(2013)409-425.

[19] J. Lei, M. Mackey, Stochastic differential delay equation, moment stability, and application to hematopoitic stem cell regulation systems, SIAM J. Appl. Math. 67(2)(2007)387-407.

[20] M. Li, Q. Deng, Almost sure stability with general decay rate of neutral stochastic delayed hybrid systems with Lévy noise, Nonlinear Anal-Hybri. Syst. 24(2017)171-185.

[21] X. Li, Q. Zhu, D. O'Reganc, pth moment exponential stability of impulsive stochastic functional differential equations and application to control problems of NNs, Journal of the Franklin Institute 351 (2014), 4435-4456.

225 [22] R.Sh. Lipster, A.N. Shiryayev, Theory of Martingales, Kluwer Academic Publisher, 1989.

[23] J. Liu, On asymptotic convergence and boundedness of stochastic systems with time-delay, Automatica 48(2012)3166-3172.

[24] X. Mao, Stochastic Differential Equations and Their Applications, 2nd Edition, Chichester: Horwood Pub., 2007. 
[25] F. Jiang, Y. Shen, J. Hu, Stability of the split-step backward Euler scheme for stochastic delay integro-differential equations with Markovian switching, Commun. Nonlinear Sci. 16(2011)814-821.

[26] X. Mao, Razumikhin-type theorems on exponential stability of stochastic functional differential eqautions, Stoch. Proc. Appl. 65(1996)233-250.

[27] X. Mao, Exponential stability of stochastic delay interval systems with Markovian switching, IEEE Trans. Automat. Control 47(10)(2002)1604-1612.

28] X. Mao, J. Lam, L. Huang, Stabilisation of hybrid stochastic differential equations by delay feedback control, Systems \& Control Letters 57(2008)927-935.

[29] X. Mao, C. Yuan, Stochastic Differential Equations with Markovian Switching, Imperial College Press, 2006.

[30] S.-E.A. Mohammed, Stochastic Functional Differential Equations, Longman Scientific and Technical, 1984.

[31] A. Rathinasamy, M. Balachandran, Mean-square stability of semi-implicit Euler method for linear stochastic differential equations with multiple delays and Markovian switching, Appl. Math. Comput. 206(2008)968-979.

[32] A. Rathinasamy, M. Balachandran, Mean-square stability of Milstein method for linear hybrid stochastic delay integro-differential equations, Nonlinear Anal.-Hybri. Syst. 2(2008)1256-1263.

[33] M. Reiss, Adaptive estimation for affine stochastic delay differential equations, Bernoulli 11(1)(2005)67-102

[34] M. Shen, W. Fei, X. Mao, S. Deng, Exponential stability of highly nonlinear neutral pantograph stochastic differential equation, Asian Journal of Control, in press.

[35] M. Shen, W. Fei, X. Mao, Y. Liang, Stability of highly nonlinear neutral stochastic differential delay equations, Systems \& Control Letters 115(2018)1-8.

[36] J. Tan, H. Wang, Convergence and stability of the split-step backward Euler method for linear stochastic delay integro-differential equations, Math. Comput. Model. 51(2010)504-515.

37] X. Wu, Y. Tang, W. Zhang, Stability analysis of stochastic delayed systems with an application to multi-agent systems, IEEE Trans. Automat. Control 61(12) (2016)4143-4149.

[38] X. Wu, Y. Tang, J. Cao, X. Mao, Stability analysis for continuous-time switched systems with stochastic switching signals, IEEE Trans. Automat. Control, DOI 10.1109/TAC.2017.2779882.

[39] S. Xu, J. Lam, X. Mao, Delay-dependent H-infinity control and filtering for uncertain Markovian jump systems with time-varying delays, IEEE Transactions on Circuits and Systems I 54(9)(2007)2070-2077.

[40] S.R. You, W. Liu, J.Q. Lu, X. Mao, J.W. Qiu, Stablization of hybrid systems by feedback control based on discretetime state observation, SIAM J. Control Optim. 53(2)(2015)905-925.

[41] D. Yue, Q. Han, Delay-dependent exponential stability of stochastic systems with time-varying delay, nonlinearity, and Markovian switching, IEEE Trans. Automat. Control 50(2005)217-222. 